\title{
The teapot effect: sheet-forming flows with deflection, wetting and hysteresis
}

\author{
By S. F. KISTLER † AND L. E. SCRIVEN \\ Department of Chemical Engineering and Materials Science, University of Minnesota, \\ Minneapolis, MN 55455, USA
}

(Received 5 February 1990 and in revised form 3 April 1991)

The flow of a two-dimensional viscous film falling from the edge of an inclined plane exhibits a distinctive set of phenomena which, in various combinations, have been referred to as the teapot effect. This paper makes plain that three basic mechanisms are at the root of these phenomena: deflection of the liquid sheet by hydrodynamic forces, contact-angle hysteresis, and multiple steady states that give rise to a purely hydrodynamic hysteresis. The evidence is drawn from Galerkin/finite-element analysis of the Navier-Stokes system, matched to a one-dimensional asymptotic approximation of the sheet flow downstream, and is corroborated experimentally by flow visualization and measurements of free-surface profiles and contact line position. The results indicate that the Gibbs inequality condition quantifies the inhibiting effect of sharp edges on spreading of static contact lines, even in the presence of flow nearby. The branchings, turning points, and isolas of families of solutions in parameter space explain abrupt flow transitions observed experimentally, and illuminate the stability of predicted flow states.

\section{Introduction}

This paper reports a comprehensive study of two-dimensional sheet-forming flows: liquid falls as a sheet, or curtain, from the bottom edge, or lip, of an inclined plate down which runs a film, as shown in figure 1 . This free-surface flow is central in curtain coating where an unsupported liquid sheet falls under the action of gravity onto a moving substrate being coated (Greiller 1972). Understanding this flow is also relevant to many other coating and polymer processing flows because it incorporates a common feature, a static contact line where a free surface separates from the solid surface. The spontaneous advance, retreat, and seeming attachment of static contact lines at sharp edges can critically influence the uniformity of the sheet or coating produced. Beyond practical aspects, the flow in figure 1 is a fluid mechanical curiosity. It exhibits a peculiar set of phenomena that are all too familiar from everyday occurrence, and have evoked numerous scientific explanations (Reiner 1956, 1969; Keller 1957; Harlow \& Amsden 1971; Lin \& Krishna 1978; Walker 1984; Vanden-Broeck \& Keller 1986, 1988).

Figure 2 illustrates these phenomena. Liquid issuing rapidly from an inclined spout arches downward along a ballistic-like trajectory (figure $2 a$ ). As the flow rate diminishes, the stream bends back more and more toward the underside of the spout $(b-d)$. Figure 2(e) shows how the stream can make contact with the underside of the spout, wet it, and dribble some way down it before falling off. Thus wetting and spreading phenomena can enter the picture, and they can shift drastically the line of

$\dagger$ Present address: 3M Company, 236-1N-05 3M Center, St. Paul, MN 55144-1000, USA. 

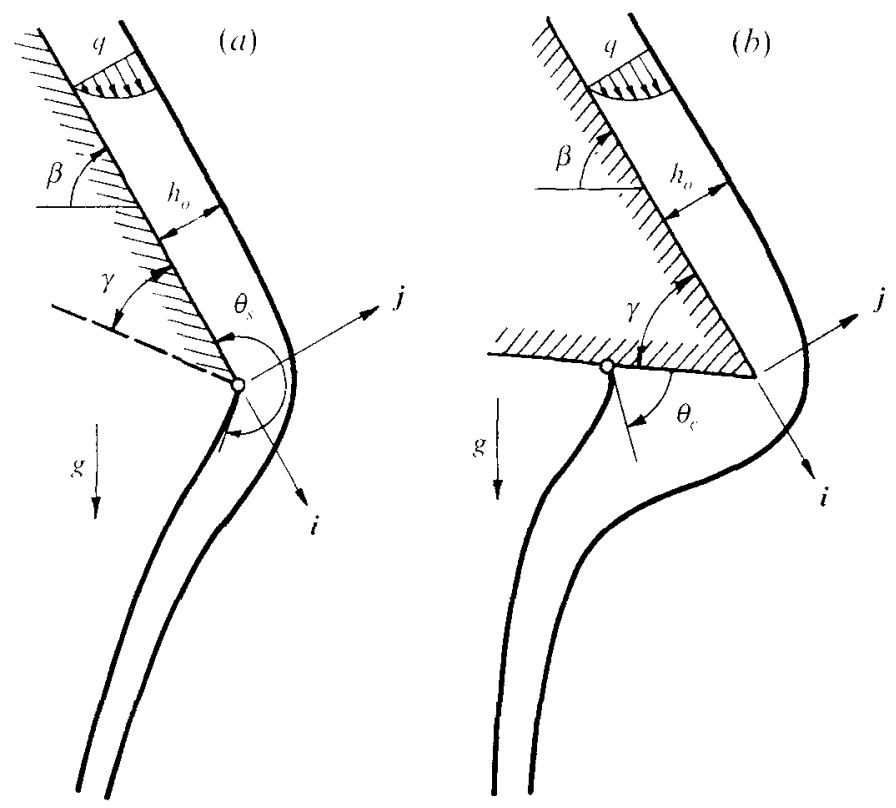

Figure 1. A two-dimensional viscous film falling from the lip of an inclined plate to form an unsupported liquid sheet, or curtain: $(a)$ contact line pinned: $(b)$ contact line free to migrate.

(a)
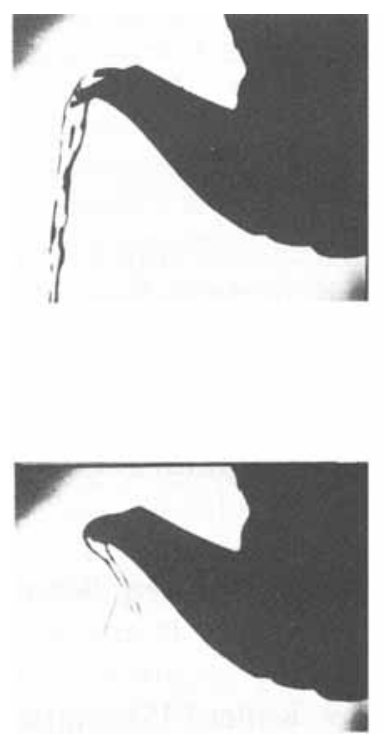

(d) (b)
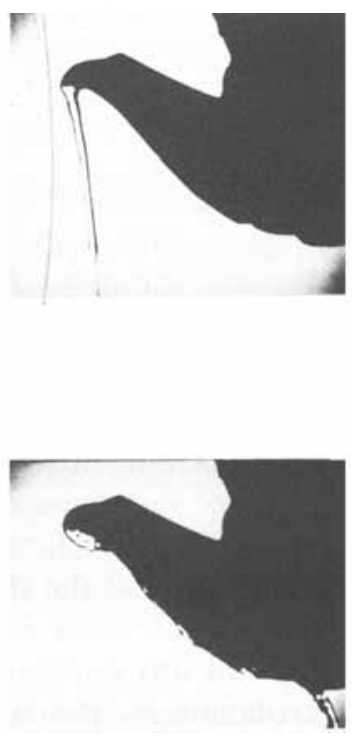

(e) (c)
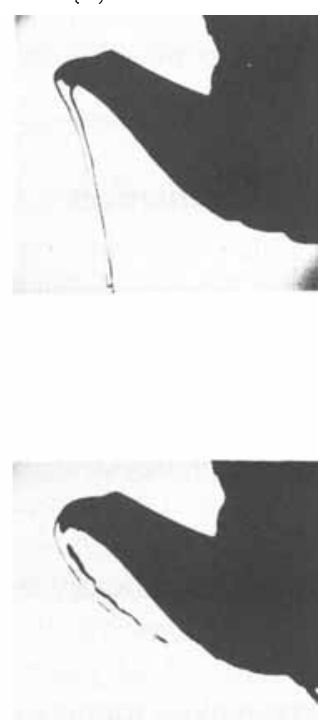

$(f)$

Figurf 2. Photographs of a water stream flowing from the spout of a teapot. The flow rate is decreased from $(a)$ to $(e)$, and increased from $(e)$ to $(f)$.

three-phase contact where the liquid detaches from the solid. Figure $2(f)$ shows how the liquid can persist in wetting the solid surface when the flow rate is again increased: a septum of flowing liquid connects the main stream to the now pre-wetted spout. Evidently there is hysteresis with respect to flow rate.

This set of phenomena is often called the teapot effect, a usage reinforced by Reiner 
(1956, 1969) who was the first to suggest an explanation of the physical mechanisms at work. He distinguished two flow phenomena. One is the liquid layer flowing in contact with the wall in such a way, Reiner held, that 'vortices' are generated which keep the liquid 'pressed' against the wall. The second phenomenon Reiner called the 'anti-ballistic' jet, but left unexplained. Keller (1957) offered an explanation in terms of two-dimensional potential flow theory from which the actions of viscosity, surface tension, and gravity were excluded. In that approximation, the speed of the liquid rises and its pressure falls at the edge, producing across the film a pressure difference which forces the stream to flow around the edge. Using a Marker-and-Cell (MAC) finitedifference scheme, Hirt \& Shannon (1968) simulated the flow of a viscous liquid layer falling off the lip of an inclined plane. They attributed the anti-ballistic action to a cross-flow pressure gradient caused by the singularity at the sharp edge. This led some to regard the teapot effect as a relative of the Coanda effect by which a jet may attach to a wall (Harlow \& Amsden 1971; see also Martin \& Friedman 1974). Lin \& Krishna 1978 ) invoked macroscopic mass and momentum balances, accounting for inertia, gravity, viscous, and surface tension effects. However, they had to make several ad hoc assumptions about the flow field near the lip, and introduced an empirical parameter $\left(h_{l}\right)$ to estimate the local film thickness where the liquid sheet forms. They concluded that the sheet deflects more as surface tension and viscosity rise, and that it can fall vertically only when the liquid is inviscid. These conclusions, however, agree with neither the theory nor the measurements presented below. Walker (1984) recounted Reiner's and Keller's explanations and reported some additional experiments, but did not mention the hysteresis phenomenon shown in figure 1. Pritchard (1986) published experimental observations of liquid being poured over the end of a plate inclined at a small angle. He considered a lip configuration different from the one studied here, and focused on classifying a wide range of different, mostly unstable, flow configurations. For some flows in which the liquid formed a stable sheet, he uncovered multiple flow states at the same flow rate (see his figure 4), but offered no explanation. VandenBroeck \& Keller (1986) extended the original potential-flow analysis (Keller 1957) to account for gravity. In addition to film-like flows which adhere to the underside of the lip, they analysed sheet-like flows with two free surfaces, one separating from the solid at the sharp edge of the lip. More recently, they also allowed for film flows that first adhere to the solid, but then separate from the lip underside, forming a second free surface at a detachment line away from the lip (Vanden-Broeck \& Keller 1988). These potential-flow analyses, however, do not account for viscous and surface tension effects. Like previous attempts to explain the teapot effect, they cannot resolve all the phenomena observed in experiments.

The present work follows neither Reiner's arguments nor those that succeeded them, but presents a fresh view of the experiment pictured in figure 2, and of experiments with the closely related two-dimensional flows shown in figure 1. From these experiments it appears that three basic mechanisms are at the root of the teapot effect. First is a purely hydrodynamic deffection of the liquid stream. Second is contact-angle hysteresis of the sort recognized in surface chemistry (cf. Johnson \& Dettre 1969; Adamson 1982), and restricted contact line advance and retreat at a sharp edge that make the contact line seem pinned (see Oliver, Huh \& Mason 1977). Third are multiple steady flow states and free-surface configurations that are sometimes possible at the same contact angle.

The objectives of our study are to predict the set of phenomena that constitutes the teapot effect from a complete analysis of the Navier-Stokes system for incompressible, viscous free-surface flow, and to corroborate those predictions by systematic experiments. The effect as seen in pouring tea from a pot is three-dimensional and a 
formidable challenge to fluid mechanical analysis. Essentially the same phenomena can be seen, however, in the two-dimensional version of the teapot effect analysed here: refer to figure 1. Even for this flow, the analysis faces two major challenges. First, the free surfaces are quite free, especially when the contact line is not pinned at the sharp edge: not only the details of meniscus shape, but also the entire flow domain can change shape severely as flow parameters vary. Second, there is no obvious downstream boundary condition at any prospective outflow plane a short distance downstream of the lip. The sheet thickness and inclination are unknown, and the flow rearrangement that continues downstream defies a simple statement about the stress distribution in the sheet. Only far enough downstream that viscous effects have become unimportant does the flow approach asymptotically a state of free gravity fall. The first challenge is met here by an approach developed for the purpose (Kistler \& Scriven 1983, 1984): the Navier-Stokes system is solved by Galerkin's method with convenient finite-element basis functions and so-called isoparametric mapping of the flow domain into a rectangular strip. Central in this approach is a flexible parametrization of the free surfaces which, together with the division of the flow domain into sub-domains, or finite elements, can cope with highly irregular flow domains. The parametrization is based on generator lines, or spines, that translate and rotate adaptively and automatically so as to accommodate changes in contact line position and sheet deflection. The second challenge is met by dividing the flow into two zones (see figure 3): a sheet-forming zone around the lip where the liquid changes direction and falls off the inclined plane, acquiring a second free surface which separates from a static contact line; and a sheet-flow zone beyond the lip where the falling liquid sheet approaches a virtually pure extensional flow regime in which acceleration by gravity contends with upstream influence through normal viscous stress that diminishes and asymptotically vanishes downstream. Solutions of one-dimensional asymptotically valid equations that describe the flow in the falling sheet-flow zone downstream are matched to twodimensional solutions of the complete Navier-Stokes system in the sheet-forming zone upstream.

In our experiments, liquid sheets falling from the lip of an inclined plane were produced with a simple table-top apparatus. An optical sectioning technique with a sheet of laser light was developed to visualize film and sheet profiles. An adaptation of that technique was used to locate contact lines. A mechanical micrometer-needle probe was devised to measure precisely the shape of free surfaces.

Section 2 states the Navier-Stokes system for two-dimensional sheet-forming flows, puts forward the asymptotic equations for the one-dimensional sheet-flow downstream, and establishes the conditions of matching those two flow representations. Section 3 highlights the Galerkin/finite-element solution procedures that divide the domain into sub-domains, or elements, expand the dependent variables in terms of a set of simple polynomial basis functions, reduce the continuity and momentum principles to discretized algebraic equations for the coefficients of the basis functions, and solve the resulting nonlinear equation set by Newton iteration, with initialization by continuation. Section 4 describes the flow apparatus and measurement techniques, and recounts the experimental procedures.

Thereafter, the computer-generated, theoretical predictions and the experimental observations are divided as follows. Section 5 contains those results in which the contact line remains pinned at the sharp lip of the inclined plane. In this case, there is purely hydrodynamic deflection that can be traced to the lack of shear in the freefalling sheets as compared to the film flow on the inclined plane. Section 6 reports those results in which the contact line takes up a position on the underside of the lip, away 


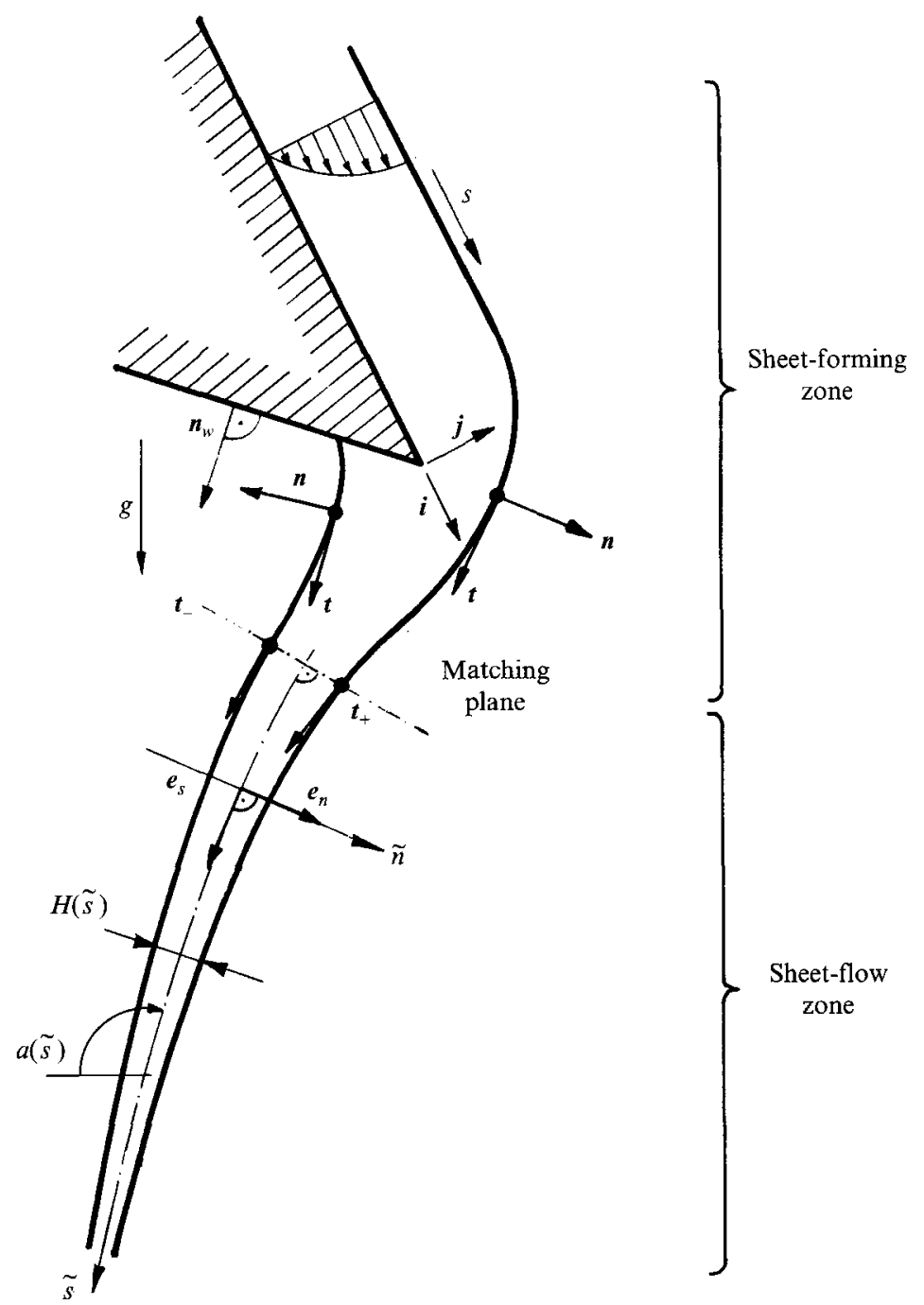

FIGURE 3. Analysis by zones of viscous sheet-forming flows.

from the lip's edge. In this case, the Gibbs inequality condition is violated ( $\$ 6.1)$. The results suggest that the contact-angle hysteresis commonly seen in static menisci with contact lines is evidently the same when liquid is flowing at an appreciable rate (\$6.2). The analysis also uncovers multiple steady states that arise from the nonlinearities of the Navier-Stokes system and the free boundary conditions ( $\$ 6.3)$. These multiple steady states lie on solution branches in parameter space that are connected at turning points where abrupt flow transitions give rise to a purely hydrodynamic hysteresis. This previously unrecognized hysteresis is quite distinct from the contact-angle hysteresis that is commonly associated with surface roughness and heterogeneity, but might also result from delayed equilibration of three-phase contact regions. Graphs of solution branches and isolas $(\S 6.4)$, with their turning points, bring out the structure of the parameter space (Reynolds number, property parameter, contact angle, inclination angle of the plane, and cut-back angle of the lip). The stability of particular steady states, even though only to a limited class of disturbances, is related directly to their location with respect to the turning points in the parameter space. 
In $\S 7$, the implications of the results are discussed in regard to the methods used, and the mechanisms uncovered. The consequences of the teapot effect are as undesirable when pouring tea from a pot as they are in modern high speed precision curtain coating (Raux 1976). Once complete physical understanding is at hand, it can be used to advantage in designing lip configurations that prevent the deleterious effect. It can also suggest how to vary flow parameters in start-up so as to avoid the operating hysteresis stemming from the multiple steady states.

\section{Governing equations}

\subsection{Two-dimensional sheet-forming flow}

In the sheet-forming zone of figure 1, the pointwise momentum and mass conservation equations in dimensionless form are

$$
\begin{gathered}
\operatorname{Re} \boldsymbol{v} \cdot \boldsymbol{\nabla} \boldsymbol{v}=\boldsymbol{\nabla} \cdot \boldsymbol{T}+3(\sin \beta \boldsymbol{i}-\cos \beta \boldsymbol{j}) / \sin \beta, \\
\boldsymbol{\nabla} \cdot \boldsymbol{v}=0 .
\end{gathered}
$$

Velocity, which is expressed as $v=u i+v j$, is measured in units of $U=q / h_{o}=$ $\left(\rho g q^{2} \sin \beta / 3 \mu\right)^{\frac{1}{3}}$, the average velocity in the fully developed film on the inclined plane. Length is measured in units of $h_{o}=(3 \mu q / \rho g \sin \beta)^{\frac{1}{3}}$, the thickness of that film. Here, $i$ and $j$ are the unit vectors in the directions parallel and normal to the inclined plane; $q$ is the pre-metered volumetric flow rate per unit width (i.e. perpendicular to the section in figure 1$) ; \beta$ is the inclination angle of the plane with respect to horizontal; $\rho$ is the liquid density; $\mu$ is the liquid viscosity; and $g$ is the gravitational acceleration. $\boldsymbol{T}$ is the stress tensor, here taken to be that of incompressible Newtonian liquid, namely $\boldsymbol{T}=-p \boldsymbol{I}+\left[\boldsymbol{\nabla} \boldsymbol{v}+\boldsymbol{\nabla} \boldsymbol{v}^{T}\right]$, where $p$ is pressure and $\boldsymbol{I}$ is the unit tensor. Both $\boldsymbol{T}$ and $p$ are measured in units of $\mu U / h_{0}$. Re $\equiv \rho q / \mu$ is the Reynolds number.

The assumption of two-dimensional flow implies that a large central portion of the curtain flow remains undisturbed by so-called edge guides which are needed to maintain a specified width of the falling curtain (cf. Lin 1981). They typically are vertical rods mounted near the lip (e.g. Greiller 1972). Two-dimensionality also implies that there are no variations in the transverse direction that are caused, for instance, by uneven wetting of the lip underside, or by flow instabilities. Pritchard (1986) gave a detailed account of various instabilities in sheet-forming flows. These instabilities, however, occur at lower flow rates than are of interest in this paper.

At some inflow plane $x=x_{I}$ sufficiently far upstream, the essential velocity boundary condition is that of rectilinear film flow:

$$
v=1.5\left(2 y-y^{2}\right) i \text {. }
$$

The dimensionless film thickness at $x=x_{I}$ is unity. At the solid boundaries there is neither slip nor penetration:

$$
v=\mathbf{0} .
$$

At the free surfaces, the traction condition

$$
\boldsymbol{n} \cdot \boldsymbol{T}=N_{\sigma}\left[768 /\left(R e^{2} \sin \beta\right)\right]^{\frac{1}{8}} \mathrm{~d} \boldsymbol{t} / \mathrm{d} s-\boldsymbol{n} \boldsymbol{p}^{A}
$$

relates the normal stress in the liquid to the capillary pressure, and also requires the shear stress to vanish; the kinematic boundary condition

$$
\boldsymbol{n} \cdot \boldsymbol{v}=\mathbf{0}
$$

expresses that there be no mass flow through the interface. Here $t$ is the unit tangent vector pointing in the direction of increasing distance $s$ along the surface (cf. figure 3 ), 
$\boldsymbol{n}$ the outward pointing unit normal, and $p^{A}$ the hydrostatic pressure in the ambient gas which, by assumption, exerts no shear on the liquid. The property parameter $N_{\sigma} \equiv \sigma /\left[(4 \mu)^{4} \mathrm{~g} / \rho\right]^{\frac{1}{3}}$ is preferred over the capillary number

$$
C a \equiv \mu U / \sigma=1 / N_{\sigma}\left[\operatorname{Re}^{2} \sin \beta / 768\right]^{\frac{1}{3}},
$$

which normally enters the capillary pressure term in (5), because it is independent of flow rate $q$, the primary variable in experiments (the factor $768=3 \times 4^{4}$ arises from the particular choice for $U=q / h_{o}$ and the definition of $N_{\sigma}$ ).

When the contact line is taken to be pinned at the sharp edge, the essential condition on contact line position is

$$
\boldsymbol{x}=\mathbf{0} \text {. }
$$

When the contact line is free to migrate, the apparent contact angle $\theta_{c}$ - the angle between the normal to the solid surface, $\boldsymbol{n}_{w}$ (cf. figure 3 ), and that to the visible free surface at the putative contact line, $\boldsymbol{n}_{c}$ - becomes an additional input parameter; it is specified by the boundary condition

$$
\boldsymbol{n}_{c} \cdot \boldsymbol{n}_{w}=\cos \theta_{c}
$$

Even though it is a common measure of wettability, the apparent contact angle $\theta_{c}$ is a macroscopic concept that incorporates information about submicroscopic details of 'disjoining forces' (or their potentials, the various surface excess energies), surface roughness, surface heterogeneity and contamination, and dynamic effects that can give rise to hysteresis phenomena (see Johnson \& Dettre 1969; Oliver, Huh \& Mason 1980; Mohanty 1981; and Teletzke, Davis \& Scriven 1987, 1988). Comparisons between theory and experiments in $\$ 6$ below confirm that the concept of a 'quasi-static' contact angle is a useful one even in the presence of flow. At the contact line, the change from adherence to the solid to no shear along the free surface causes a local singularity which is logarithmically weak and, hence, integrable (Michael 1958; Richardson 1970). For contact lines pinned at sharp edges, the singularity remains an inconsequential detail on the scale of macroscopic flow, and no-slip of the liquid on the solid appears to be an adequate boundary condition (e.g. Chang, Patten \& Finlayson 1979; Silliman \& Scriven 1980; Gear et al. 1983). An earlier analysis of a free-surface flow with a freely moving static contact line (Dupret 1982), as well as the results in $\$ 6$ suggest that the same treatment is appropriate also for contact lines that are free to migrate.

The chosen outflow plane is orthogonal to the sheet midsurface as indicated in figure 3 . At that plane, boundary conditions on the streamwise component of momentum flux and surface tension force and on the transverse velocity component arise from matching the two-dimensional solution of sheet-forming flow upstream to a onedimensional approximate solution of falling-sheet flow downstream. These matching conditions are described in $\$ 2.3$.

\subsection{One-dimensional approximation to falling-sheet flow}

Away from forming zones, various schemes of approximate analysis suffice to approximate the flow in thin liquid sheets, for typically variations in the streamwise velocity and in stress are small across the sheet. Such schemes can be based on integral momentum balances or perturbation analysis, as reviewed by Kistler (1984), who also put forward a pair of equations that describe the position and shape of twodimensional unsymmetrically falling liquid sheets. That pair of so-called sheet-profile equations is the one employed here. It permits a drastic reduction of the number of equations and unknowns in the finite-element analysis of the sheet-flow zone downstream of the lip, so that the computational effort can be concentrated near the lip, where it is most needed. 
The strategy behind deriving the profile equations of freely falling liquid sheets is an extension of Higgins \& Scriven's (1979) integral momentum approach for deriving equations that describe the profile of liquid films supported by a flat solid substrate. In the case of unsupported liquid sheets (for complete details, see Kistler 1984), integrating the momentum balances in directions normal and tangential to the sheet across the sheet thickness and eliminating pressure by means of the normal momentum balance and normal stress boundary condition yields a pair of coupled integrodifferential equations. These equation relate the variables of main interest, the inclination of the sheet trajectory and the sheet thickness along that trajectory, to the velocity field and viscous stress field within the sheet. With the Newtonian constitutive relation for viscous stress and a suitable approximation to the velocity field, the integrals in the integrodifferential equations can be evaluated. When the characteristic sheet thickness, $T$, is assumed to be much smaller than the characteristic distance in the streamwise direction over which the flow varies significantly, $L$, i.e. $T / L \ll 1$, and the radius of curvature of the liquid sheet is taken to be much smaller than its thickness, so that everywhere $(T / L)(\mathrm{d} \alpha / \mathrm{d} S) \ll 1$ (here $S$ is the distance along the sheet measured in units of $L$, and $\alpha(S)$ is the angle of inclination of the sheet midsurface), local plugflow in the streamwise direction adequately describes the velocity field in the sheet to leading order. These assumptions, and the resulting simplifications, are referred to as the thin-sheet flow approximation.

The result is a pair of coupled ordinary differential equations that describe the thickness profile and inclination angle along unsymmetrically falling liquid curtains (Kistler 1984):

$$
\begin{gathered}
\frac{\operatorname{Re} N_{\sigma}}{8} \frac{\mathrm{d}\left(H^{\prime \prime}-H \alpha^{\prime 2}\right)}{\mathrm{d} \tilde{s}}+\frac{H^{\prime}}{H^{3}}+\sin \alpha-\left(\frac{H^{\prime \prime}}{H^{2}}-\frac{H^{\prime 2}}{H^{3}}\right)=0, \\
\alpha^{\prime}\left(\frac{8 N_{\sigma}}{\operatorname{Re}}-\frac{1}{H}-\frac{H^{\prime}}{H}\right)+\cos \alpha H=0 .
\end{gathered}
$$

Here $H(\tilde{s})$ is the sheet thickness, measured normal to the sheet midsurface in units of $\frac{1}{4} \operatorname{Re}(4 \mu / \rho)^{\frac{2}{3}} g^{-\frac{1}{3}} ; \alpha(\tilde{s})$ is the inclination angle of the midsurface from horizontal; $\tilde{s}$ is the distance along the midsurface, measured in units of $(4 \mu / \rho)^{\frac{2}{3}} g^{-\frac{1}{3}}$; and the prime denotes differentiation with respect to $\tilde{s}$. The lengthscales chosen here are independent of the characteristic length $h_{o}$ in the sheet-forming zone: they depend solely on liquid properties and gravitational acceleration, and thus are appropriate in the downstream regime of asymptotic approach to free gravity fall. The physical origin of the terms in (9) and (10) is transparent: in the direction normal to the sheet, gravity balances the 'shell' forces arising from surface tension, normal viscous stress, and the centrifugal force; and in the tangential direction, the pressure force due to the capillary pressure gradient, the normal viscous force, inertia and gravity are in equilibrium.

The upstream boundary conditions on sheet thickness and inclination angle emerge from matching the approximate sheet profile equations to the complete equation set in the sheet-forming zone upstream: see $\$ 2.3$ below. The downstream boundary conditions describe free gravity fall:

$$
\begin{gathered}
H^{\prime}=-H^{3} \sin \alpha, \\
H^{\prime \prime}=H^{5}\left(3 \sin ^{2} \alpha-\cos ^{2} \alpha\right) .
\end{gathered}
$$

Condition (11) is the tangential momentum balance (9) specialized for the case in which inertia and gravity dominate. In deriving (12), the relationship $\alpha^{\prime}=H^{2} \cos \alpha$ has been used. The latter is the normal momentum balance (10) simplified by the assumption 
that sufficiently far downstream the centrifugal term $\alpha^{\prime} / H$ dominates the surface tension term $\alpha^{\prime} 8 N_{\sigma} / R e$. Experiments with symmetrically falling liquid curtains confirm that, at moderately high Reynolds numbers $R e \geqslant O(1)$ which are of interest in this paper, the flow approaches free gravity fall a few sheet thicknesses downstream from the nozzle where the sheet is formed (Brown 1961). For symmetrical sheets and jets, asymptotic approach to free gravity fall has also been verified with formal perturbation procedures (e.g. Clarke 1968; Joseph, Ngyen \& Matta 1983). For the non-symmetrical sheet flows analysed in this paper, the validity of the downstream boundary conditions (11) and (12) is established by demonstrating that computed predictions are insensitive to the locations $\tilde{s}$ where these conditions are imposed. In a typical solution shown in $\S 5$ below (i.e. for $\operatorname{Re}=7, N_{\sigma}=1.15, \beta=90^{\circ}$ ), lengthening the one-dimensional domain from 9.51 to 89.94 units of $(4 \mu / \rho)^{\frac{2}{3}} g^{-\frac{1}{3}}$ changed the velocity field and freesurface locations in the two-dimensional sheet-forming region by less than $0.01 \%$.

Sufficiently far downstream, of course, the liquid sheet impinges onto some solid object or plunges into a pool of liquid. However, once it has reached a state of free gravity fall, the flow cannot transmit any upstream influence: the equations become of parabolic type. Under these conditions, the details of the impingement flow - which are beyond the scope of this paper - have no effect on the sheet-forming flow. Where the sheet impinges, it may exhibit a set of standing waves analogous to those observed for impinging jets (Cullen \& Davidson 1957). The amplitude of such waves, however, decays exponentially in the upstream direction (Bretherton 1961; Ruschak 1978) and would be undetectable where the sheet forms. The sheet may also suffer a buckling instability of the sort first described by Taylor (1968). Cruickshank \& Munson (1981) established that this is a low-Reynolds-number phenomenon well outside the parameter ranges investigated here $[R e \geqslant O(1)]$. In actual curtain coaters, the falling sheet impinges on a moving substrate. Recent studies of curtain coating by Ogawa \& Scriven (1994) confirm that, above a critical curtain height, there is no coupling between the coating flow and the sheet-forming flow upstream.

\subsection{Matching conditions}

Matching the one-dimensional sheet flow with the two-dimensional main flow ensures that the distinct approximations of the flow field on either side of the open-flow boundary, here referred to as the matching boundary, comply with the fundamental physical requirements that the mass flux, the momentum flux, and the free surfaces be continuous at the matching boundary. Here the matching conditions are merely summarized. They are readily derived and Kistler (1984) gives full details.

The downstream boundary conditions that complete the mathematical description of the two-dimensional sheet-forming flow are

$$
\begin{gathered}
T_{s s} \equiv \boldsymbol{e}_{s} \boldsymbol{e}_{s}: \boldsymbol{T}=r^{2} \frac{\operatorname{Re}}{4}\left\{\frac{1}{2}\left(H^{\prime \prime}-H \alpha^{\prime 2}\right) N_{\sigma} \operatorname{Re}-\frac{4 H^{\prime}}{H^{2}}-\operatorname{Re} \frac{\tilde{n}}{H}\left(\frac{\alpha^{\prime}}{H}-\cos \alpha H\right)\right\}, \\
\frac{1}{C a} \boldsymbol{e}_{s} \cdot \boldsymbol{t}_{ \pm}=\frac{N_{\sigma}}{\operatorname{Re}\left[1+\left(H^{\prime} \operatorname{Re} / 8\right)^{2}\right]^{\frac{1}{2}}}, \\
v_{n} \equiv \boldsymbol{e}_{n} \cdot \boldsymbol{v}=r \frac{\operatorname{Re}}{4} \frac{\tilde{n}}{H} \frac{H^{\prime}}{H} .
\end{gathered}
$$

Here $e_{s}$ and $e_{n}$ are the unit vectors in the directions tangential and normal to the sheet midsurface respectively; and $\tilde{n}$ is the distance along the matching plane, measured from the midsurface in units of $\frac{1}{4} \operatorname{Re}(4 \mu / \rho)^{\frac{2}{3}} g^{-\frac{1}{3}}$. The subscripts + and - denote the upper and lower free surfaces respectively, and $r \equiv\left(12 \sin \beta / R e^{2}\right)^{\frac{1}{3}}$ is a scale factor that reflects 
the two distinct lengthscales used. Condition (13) imposes the component of the total traction normal to the matching plane, calculated from the thin-sheet flow approximation in terms of the variables that describe the sheet-flow zone. Likewise, condition (14) specifies the component of the surface tension force normal to the matching plane. Condition (15) imposes the velocity component normal to the sheet midsurface that is consistent with local plug flow. It is preferred over imposing the shear-stress profile $T_{n s} \equiv \boldsymbol{e}_{n} \boldsymbol{e}_{s}: \boldsymbol{T}$ as a natural boundary condition because shear stress cannot be estimated accurately with the thin-sheet flow approximation.

The upstream boundary conditions that are needed to solve the sheet-profile equations (9) and (10) arise from the continuity requirements for the thickness and free-surface slopes of the liquid sheet; they are

$$
\begin{gathered}
H=r^{-1} e_{n} \cdot\left(x_{+}-x_{-}\right), \\
{[\sin (\alpha-\beta) i+\cos (\alpha-\beta) j] \cdot\left(t_{+}+t_{-}\right)=0 .}
\end{gathered}
$$

Here, $x_{+}$and $x_{-}$denote the free boundary locations at the matching plane. By virtue of the plug-like velocity profile on which the thin-sheet approximation is based, condition (16) also ensures continuity of total mass flow across the matching boundary.

The strategy of solving simultaneously the equations of two-dimensional sheetforming flow upstream and one-dimensional falling-sheet flow downstream can be validated by demonstrating that the computed predictions are insensitive to the location of the matching plane (for similar strategies, see also Novy, Davis \& Scriven 1990). For instance, for a solution at $R e=7, N_{\sigma}=1.15$, and $\beta=90^{\circ}$ (shown in figure $10 \mathrm{a}$ below), the distance from the lip to the matching plane was increased in increments of 4.5 from 4.5 to 18 . The maximum change in free-surface location in the twodimensional domain was below $1 \%$; the computed values of separation angle $\theta_{s}$ (cf. figure 1) changed less than $2 \%$; and $H=0.15646$ and $\alpha=1.6627\left(95.31^{\circ}\right.$ ) calculated from the two-dimensional finite-element solution at the matching plane located a distance of 18 film thicknesses from the lip agreed closely with $H(s)=0.15665$ and $\alpha(s)=1.6635\left(95.27^{\circ}\right)$ obtained at the same location from the one-dimensional falling-sheet equations when the matching plane was located a distance 4.5 from the lip.

\section{Finite-element solution procedures}

\subsection{Free-surface parametrization and domain tessellation}

The set of equations that governs sheet-forming flows is nonlinear and thus requires an iterative solution procedure. The main nonlinearity is due to the free surfaces, the locations of which are unknown a priori, and at which the nonlinear boundary conditions (5) and (6) apply. Additional nonlinearities come from the convective momentum term in (1), the sheet profile equations (9) and (10), and the matching conditions (13)-(17). The key to handling these nonlinearities is a scheme that reliably and efficiently readjusts the free surfaces and associated finite-element tessellation at each iteration step.

In the analysis put forward here, the free surfaces were parametrized along generators called spines (Kistler \& Scriven 1983, 1984). Spines are conveniently located and oriented lines, as shown in figure 4 . Each is defined by the coordinates $x_{B}^{i}$ of its base point and the unit vector $\boldsymbol{e}^{i}$ pointing in its direction. The nodal coordinates $\boldsymbol{x}^{k}$ of node $k(i, j)$ on the $i$ th spine are

$$
\boldsymbol{x}^{k}=\boldsymbol{x}_{B}^{i}+\left[h_{-}^{i}+w^{j}\left(h_{+}^{i}-h_{-}^{i}\right)\right] \boldsymbol{e}^{i},
$$




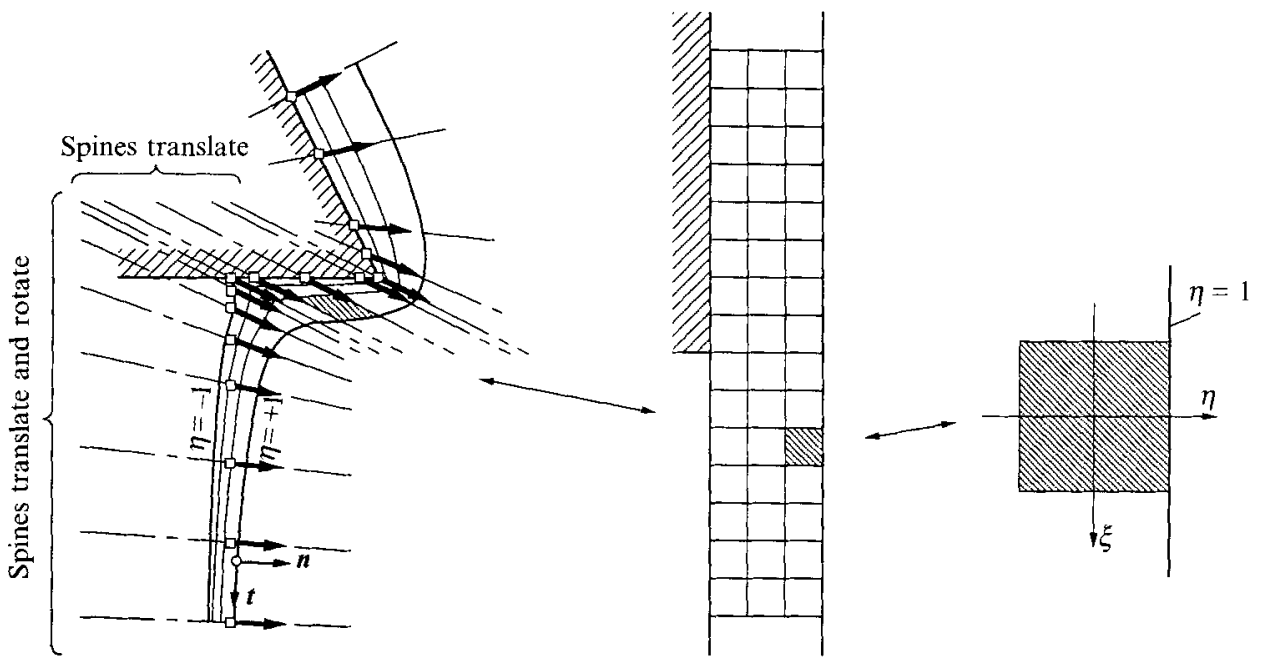

FiguRE 4. Parametrization of free surfaces and associated finite-element tessellation by means of adaptively rotating and translating spines, shown here for a case in which liquid wets the lip underside.
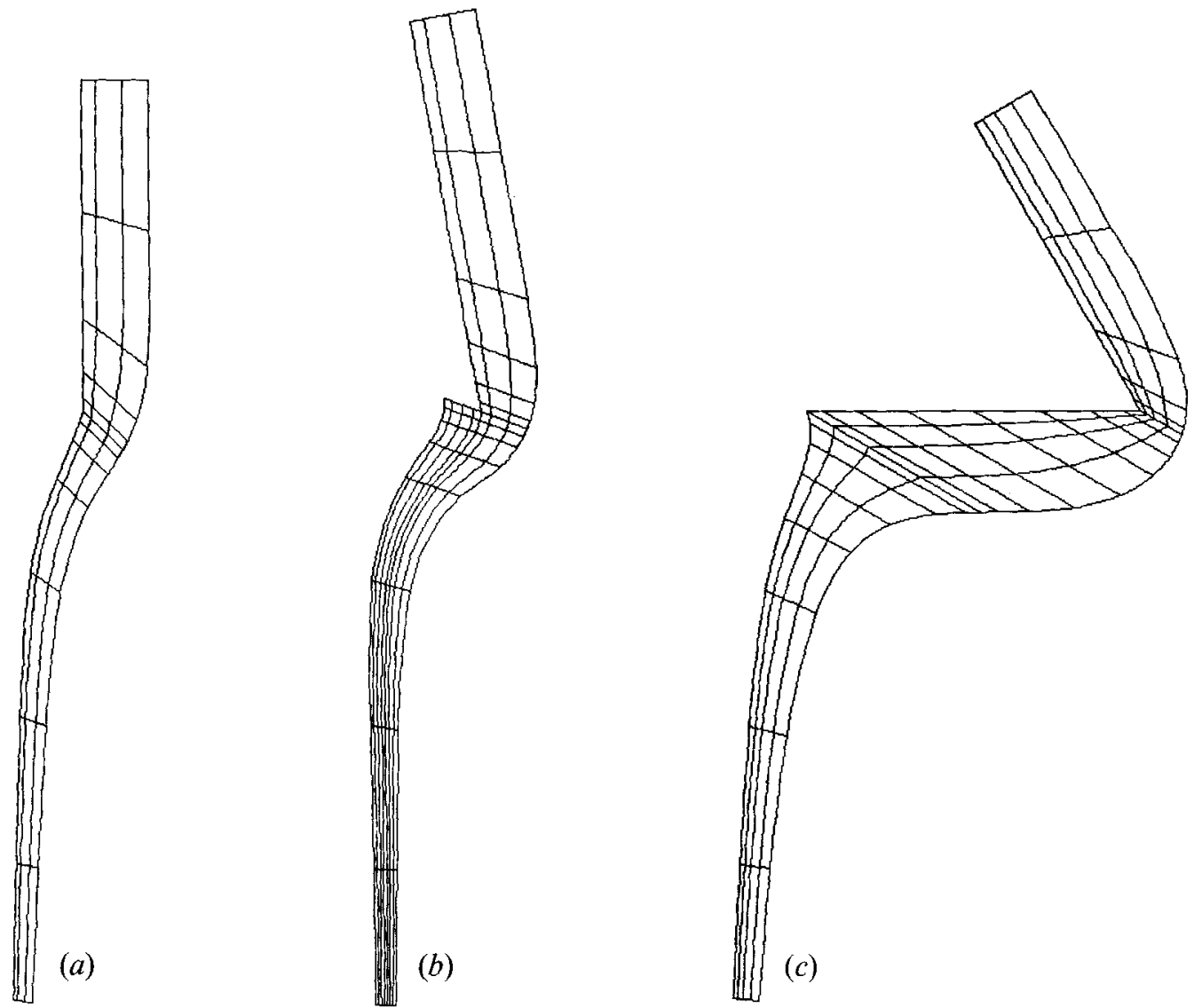

FIGURE 5. Typical finite element tessellations: (a) type A - no wetting ( $\left.R e=5, N_{\sigma}=1.15, \beta=90^{\circ}\right)$; (b) type B - slight wetting (Re $\left.=5, N_{\sigma}=1, \beta=80^{\circ}, \gamma=60^{\circ}, \theta_{c}=70^{\circ}\right)$; (c) type $\mathrm{C}$ - substantial wetting $\left(\operatorname{Re}=8, N_{\sigma}=1, \beta=60^{\circ}, \gamma=60^{\circ}, \theta_{c}=67^{\circ}\right)$. 
where $h_{+}^{i}$ and $h_{-}^{i}$ are local coordinates of the inner and outer free surfaces, unknowns to be solved for. In close conjunction with spine placement, the flow domain in the sheet-forming zone was tessellated into quadrilateral elements as indicated in figure 4 . Two opposing sides of each element were defined by spines, and thus were straight. The other two sides were made to intersect those spines at fixed relative locations $\left(w^{j}\right)$, and thus reflected the curved shape of the free surfaces. In the sheet-flow zone downstream (not shown in figure 4), the elements were one-dimensional, and were simply line segments along the sheet midsurface.

Some of the spines were made to translate and rotate adaptively so as to follow salient features of the solution: the angle of inclination $\alpha_{M}$ of the sheet midsurface at the matching plane and, in cases with wetting of the lip underside, the distance $\boldsymbol{x}_{*}$ between the contact line and the sharp edge of the lip (see Kistler 1984 for more details). The base points $x_{B}^{i}$ and the directions $e^{i}$ became functions of these additional, unknown variables. Depending on the contact line position, the finite-element tessellation was one of the three types displayed in figure 5: type A was used for solutions in which the contact line remained pinned, type B for those in which the liquid wetted the lip underside slightly, and type $C$ for those in which the liquid flowed along the lip underside over a sizable distance before falling off as a sheet.

\subsection{Basis function expansions and weighted residual equations}

In the sheet-forming zone, the unknown velocity and pressure fields were expanded in the forms

$$
\boldsymbol{v}=\sum_{k=1}^{K} \boldsymbol{v}_{k} \Phi^{k}(\xi, \eta), p=\sum_{l=1}^{L} p_{l} \Psi^{l}(\xi, \eta) .
$$

Similarly, in the sheet-flow zone, the unknown sheet thickness and inclination were expanded in the forms

$$
H=\sum_{m=1}^{M} H_{m} \chi^{m}(\xi), \quad \alpha=\sum_{n=1}^{N} \alpha_{n} \zeta^{n}(\xi) .
$$

Here, nine-node biquadratic basis functions $\Phi^{k}$ were employed for $v$, four-node bilinear ones $\Psi^{l}$ for $p$, Hermite cubic ones $\chi^{m}$ for $H(s)$, and linear ones $\zeta^{n}$ for $\alpha(s)$. The choice for $v$ and $p$ is one of the so-called mixed interpolations that should be used in velocity-pressure formulations of the Navier-Stokes system (e.g. Huyakorn et al. 1978). In the computations reported in this paper, the numbers of unknown coefficients were $203<K<387,60<L<110, M=42$, and $N=21$.

The key to expanding the free-surface shapes in the forming zone is the isoparametric mapping

$$
\boldsymbol{x}=\sum_{k=1}^{K} \boldsymbol{x}_{k}(\boldsymbol{h}) \Phi^{k}(\xi, \eta)
$$

This is an element-by-element transformation between the actual flow domain and replicates of the unit square in $(\xi, \eta)$-coordinates (cf. figure $4 ; \xi$ and $\eta$ here are local coordinates within a finite element, but they also can be interpreted as global coordinates, modulo element indices, in a computational domain). In (21), $\boldsymbol{h}$ denotes the entire set of free-surface parameters, namely $h_{+}^{i}, h_{-}^{i}, \alpha_{M}$, and $x_{*}$. Along free surfaces, the local mappings

$$
\boldsymbol{x}=\sum_{f=1}^{F} \boldsymbol{x}_{f}(\boldsymbol{h}) \Phi^{f}(\xi, \eta= \pm 1),
$$


by the biquadratic basis functions, make the curved free-surface segments simple onedimensional mappings of straight lines $(\eta= \pm 1)$ in the $(\xi, \eta)$-plane $(F$ denotes the subset of basis functions $\Phi^{f}$ that are non-zero along one of the free boundaries). As an added benefit, the unit tangent and normal vectors $t$ and $n$ along the free boundaries (where $\eta$ is either +1 or -1 ) take the computationally convenient forms

$$
\boldsymbol{t}=\frac{x_{\xi} i+y_{\xi} j}{\left(x_{\xi}^{2}+y_{\xi}^{2}\right)^{\frac{1}{2}}}, \quad n=\frac{-y_{\xi} i+x_{\xi} j}{\left(x_{\xi}^{2}+y_{\xi}^{2}\right)^{\frac{1}{2}}}
$$

Here, the subscript $\xi$ denotes differentiation with respect to that variable.

In the two-dimensional sheet-forming zone, the two scalar components of the weighted momentum residuals were required to vanish,

$$
\boldsymbol{R}_{M}^{k} \equiv \int_{A(\boldsymbol{h})}\left\{\Phi^{k}\left[\operatorname{Re} \boldsymbol{v} \cdot \boldsymbol{\nabla} \boldsymbol{v}-\frac{3(\sin \beta \boldsymbol{i}-\cos \beta j)}{\sin \beta}\right]+\nabla \Phi^{k} \cdot \boldsymbol{T}\right\} \mathrm{d} A-\oint_{s(\boldsymbol{h})} \boldsymbol{\Phi}^{k} \boldsymbol{n} \cdot \boldsymbol{T} \mathrm{d} s=0
$$

and thereby provided $2 K$ equations, as many as there were unknown velocity coefficients $u_{k}$ and $v_{k}$ (here $A(\boldsymbol{h})$ refers to the flow domain and $s(\boldsymbol{h})$ to the flow boundary). Setting the weighted residual of the continuity equation to zero,

$$
R_{C}^{l} \equiv \int_{A(h)} \Psi^{l} \nabla \cdot v \mathrm{~d} A=0
$$

provided $L$ equations, as many as there were pressure coefficients $p_{l}$, and setting the weighted residuals of the kinematic boundary condition to zero,

$$
R_{K}^{f} \equiv \int_{s_{F}(h)} \Phi^{f}(\xi, \eta= \pm 1) n \cdot v \mathrm{~d} s=0
$$

provided as many equations as there were free-surface coefficients in $\boldsymbol{h}$ (here $s_{F}(\boldsymbol{h})$ refers to a free boundary). Likewise, in the one-dimensional sheet-flow zone, the coefficients $H^{m}$ and $\alpha^{n}$ were determined by requiring that the residuals of the sheet profile equations (9) and (10), weighted with $\chi^{m}$ and $\zeta^{n}$ respectively, vanish:

$$
\begin{gathered}
\left.R_{S}^{m} \equiv \chi^{m} \frac{\operatorname{Re} N_{\sigma}}{8}\left(H^{\prime \prime}-H \alpha^{\prime 2}\right)\right|_{0} ^{\tilde{s}_{o}} \\
-\frac{\operatorname{Re} N_{\sigma}}{8} \int_{0}^{\tilde{s}_{o}}\left\{\left(H^{\prime \prime}-H \alpha^{\prime 2}\right) \frac{\mathrm{d} \chi^{m}}{\mathrm{~d} \tilde{s}}-\left[\frac{H^{\prime}}{H^{3}}+\sin \alpha-\left(\frac{H^{\prime \prime}}{H^{2}}-\frac{H^{\prime 2}}{H^{3}}\right)\right] \chi^{m}\right\} \mathrm{d} \tilde{s}=0, \\
R_{N}^{n} \equiv \int_{0}^{\tilde{s}_{o}}\left\{\alpha^{\prime}\left[2 N_{\sigma}-\frac{\operatorname{Re}}{4} \frac{1+H^{\prime}}{H}\right]+\frac{\operatorname{Re}}{4} H \cos \alpha\right\} \zeta^{n} \mathrm{~d} \tilde{s}=0 .
\end{gathered}
$$

Here $\tilde{s}_{o}$ is the arclength from the matching plane to the outflow boundary at which (11) and (12) were imposed.

Essential, or Dirichlet, boundary conditions on the velocities (3), (4) and (15), on film thickness at the inflow, and on sheet thickness (16) and sheet inclination (17) at the matching plane were imposed by substituting those conditions in place of the corresponding residual equations. Natural, or Neuman, boundary conditions, or boundary conditions of the third kind (Robin), were imposed through the boundary integral of $\boldsymbol{n} \cdot \boldsymbol{T}$ in (24), the result of applying the divergence theorem to the stress term, and through the end-point term in (27), which arises from integration by parts of the 
capillary pressure-gradient term (Kistler \& Scriven 1983, 1984). For example, the normal traction at the matching plane was imposed by substituting its one-dimensional approximate value (13) into the boundary integral in (24). Along the free surfaces, the traction condition (5) was substituted in the boundary integral in (24). After integration by parts (cf. Ruschak 1980) and with $p^{A}=0$ as pressure datum, the boundary contribution to the momentum residuals became

$$
-\int_{s_{F^{\prime}}(\boldsymbol{h})} \Phi^{k} \boldsymbol{n} \cdot \boldsymbol{T} \mathrm{d} s=\frac{1}{C a}\left\{\int_{s_{F^{*}}(\boldsymbol{h})} \boldsymbol{t} \frac{\mathrm{d} \Phi^{k}}{\mathrm{~d} s} \mathrm{~d} s-\Phi^{k}\left[\boldsymbol{t}_{\boldsymbol{1}}-\boldsymbol{t}_{\mathbf{0}}\right]\right\} .
$$

This form also permitted the conditions (14) on the directions of the surface tension forces to be imposed by direct substitution in the momentum residual equations.

\subsection{Computation of basis function coefficients}

In early finite-element analyses of viscous free-surface flows (e.g. Nickell, Tanner \& Caswell 1974), the location of a free surface had been sought by successive approximation techniques in which the free-surface location is updated on the basis of one of the boundary conditions, which condition is ignored during calculation of the flow field. Similarly, in a previously published finite-element analysis of fibre spinning in which, as in the present study, a one-dimensional approximate solution was matched to a complete two-dimensional one (Gifford 1982), the algorithm required iterating back and forth between solving the two-dimensional problem and the one-dimensional one. It is now clear, however, how to achieve a strikingly improved, quadratic rate of convergence by using Newton's method to solve, in a single iteration process, the entire set of equations for velocity, pressure, and free-surface location (Saito \& Scriven 1981; Ruschak 1980), and asymptotic sheet profiles as well (Kistler 1984).

Written in terms of a vector $\boldsymbol{b}$ of all the coefficients of the finite-element basis functions and a vector $\boldsymbol{R}(\boldsymbol{b})$ of all the weighted residuals, the Newton iteration process is, of course, the repeated solution of the linearized system of equations

$$
\boldsymbol{J}\left[\boldsymbol{b}_{n+1}-\boldsymbol{b}_{n}\right]=-\boldsymbol{R}\left(\boldsymbol{b}_{n}\right),
$$

where $\boldsymbol{J} \equiv \partial \boldsymbol{R} / \partial \boldsymbol{b}$ is the Jacobian matrix, and $n$ denotes the $n$th iteration step. The crux is the evaluation of the derivatives $\partial \boldsymbol{R} / \partial \boldsymbol{h}$ of the weighted residuals with respect to the free boundary parameters. Kistler \& Scriven $(1983,1984)$ showed that, with the spine parametrization and the isoparametric mapping, the differentiation becomes straightforward. They also explained how the full Newton system (30) of both twodimensional exact and one-dimensional approximate equations can be assembled and solved by the frontal technique (Hood 1976; Walters 1980).

A major drawback of Newton's method is its limited radius of convergence, i.e. it converges only if the initial estimate is close enough to the solution. Particularly critical is the very first approximation at the start of the sequence of parameter values of interest. Analysis of sheet-forming flows as presented here opened with an ad hoc initial guess (semi-parabolic and plug-like velocity profiles upstream and downstream of the lip respectively, and a sheet profile in accord with the limiting free gravity fall regime) for a simple case with pinned contact line, and proceeded from there to the more complicated cases with wetting of the underside of the lip. Sufficient under-relaxation, i.e. curtailed adjustment of the update $\left(\boldsymbol{b}_{n+1}=\boldsymbol{b}_{n}-\omega \boldsymbol{J}^{-1} \boldsymbol{R}\left(\boldsymbol{b}_{n}\right)\right.$, where $\left.0<\omega<1\right)$ gives the Newton iteration process the 'descent' property that will take it to a solution if there is at least one. In this study, under-relaxation was sometimes used to facilitate start-up of the computations. 
Families of sheet-forming flows were calculated by changing sequentially any of the parameters $\Pi^{T}=\left[R e, N_{r}, \beta, \gamma, \theta_{c}\right]$. Even though first-order continuation would probably have been more effective (cf. Kheshgi et al. 1983), zeroth-order continuation was used in this work, i.e. the converged solution for one set of parameters provided the initial approximation for a slightly different set. Continuation in a particular parameter fails near a turning point in that parameter, i.e. points where a solution branch turns back upon itself. Turning points appear in many of the solution families presented in $\$ 6$. In the computations, they were much in evidence owing to the narrowing of the domain of convergence as they are approached, and eventually the complete failure of Newton's method to converge. At the root of these computational difficulties were one or more Gauss elimination pivots of $\boldsymbol{J}$ which became very small as the turning was approached. At the turning point, $\boldsymbol{J}$ is singular, i.e. its determinant vanishes.

The singularity of the Jacobian matrix $\boldsymbol{J}$ at a turning point can be removed by switching continuation parameters (see e.g. Keller 1977). In this study, at a turning point in contact angle $\theta_{c}$, the continuation parameter was simply switched to the contact line position $x_{*}$, i.e. $\theta_{c}$ was made a dependent variable and $x_{*}$ was specified. At turning points in any of the other parameters $I_{j}$ in the set $\Pi$, continuation was switched to $x_{*}$ also, but instead of $\Pi_{j}$ it was again $\theta_{c}$ that was made the dependent variable. Computations were continued in that matter until the predicted angle $\theta_{c}$ was sufficiently close to the desired one that another continuation step in $\Pi_{j}$ converged to a solution on the new branch beyond the turning point. These tactics are, in essence, an ad hoc implementation of what has come to be known as Abbott's method (Abbott 1978).

\section{Experimental techniques}

\subsection{Curtain flow apparatus}

The two-dimensional teapot effect was investigated experimentally with the curtain flow apparatus shown in figure 6(a) (the numbers in the figure refer to the text that follows). Reservoir 1 contained the bulk of the approximately $0.01 \mathrm{~m}^{3}$ of waterglycerine mixtures that served as test liquids. A Zenith Model BLB-5456 gear pump, 2 , mounted on a DC motor/driver assembly with digital speed control (Zenith Products Co., West Newton, Massachusetts, USA), metered the liquid through the die, 3. On the inclined plane of that die, the liquid formed a film that fell over the sharp edge, 4 , where the freely falling curtain was formed and where the teapot effect was observed. The curtain width was maintained by two vertical edge guides, 5 , set $200 \mathrm{~mm}$ apart. These were made of stainless steel rods of $2 \mathrm{~mm}$ diameter, and touched the lip where the liquid curtain was formed. The curtain fell freely for about $150 \mathrm{~mm}$ before impinging onto the catch tray, 6 , from which the liquid returned to the reservoir, 1 . The catch tray was positioned to minimize splashing and thereby avoid bubble formation.

Details of the die design, including important dimensions, are given in figure $6(b)$. The two main components of the die were made of stainless steel and were precision ground. The film on the inclined plane was maintained at a width of $200 \mathrm{~mm}$ by strips of Teflon (1.59 mm thick). These strips caused some edge effects in the form of tiny standing waves on the film surface; these waves, however, did not affect a large central portion of the falling curtain. 


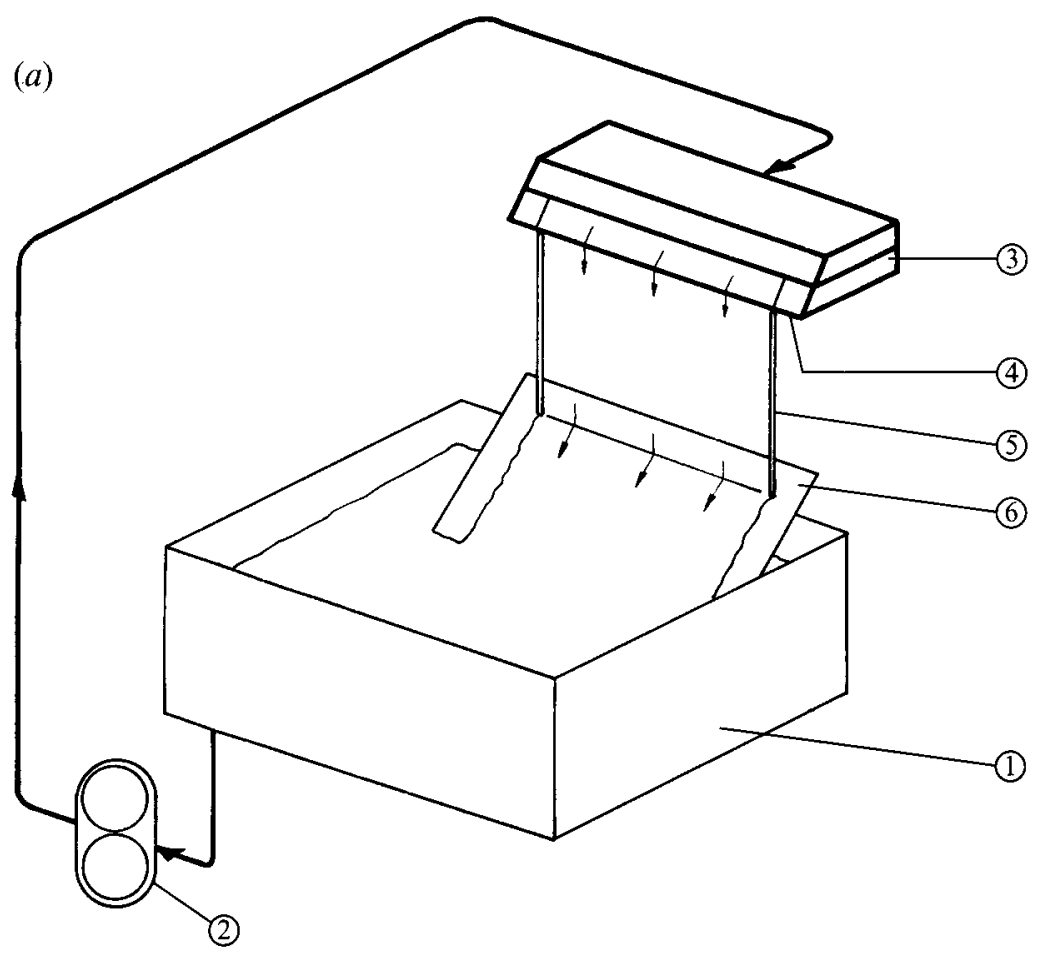

(b)

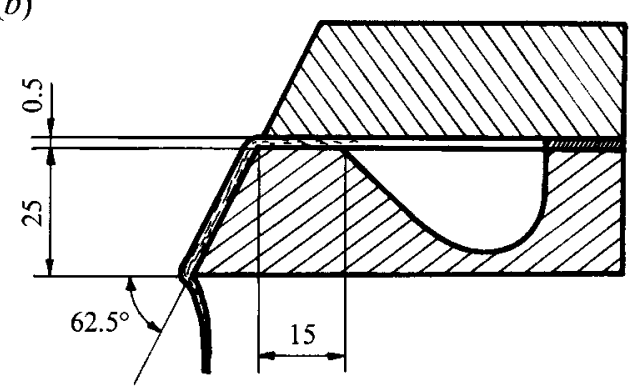

FIgure 6. Curtain flow apparatus: (a) schematic of flow system; $(b)$ die design (dimensions are in $\mathrm{mm}$ ).

\subsection{Flow visualization and measuring techniques}

Free-surface profiles in the sheet-forming zone were measured with the micrometer probe shown in figure 7. A precision micrometer $(25.4 \mathrm{~mm}$ travel, $2.54 \mu \mathrm{m}$ resolution) moved a needle tip in the $\bar{x}$-direction toward the free surface at various preset $\bar{y}$ positions. The $\bar{y}$-position was selected by means of a positioning slide equipped with a dial indicator ( $25.4 \mathrm{~mm}$ travel, $25.4 \mu \mathrm{m}$ resolution). The $\bar{x}$-position at which the needle tip just touched the free surface was readily apparent because a meniscus immediately attached to the needle. The repeatability of these measurements was within $50 \mu \mathrm{m}$. The accuracy of the probe was established by measuring the thickness of the fully developed film on the inclined plane. The deviation from the theoretical value $h_{o}$ was less than $2 \%$. The main source of error came from inaccuracies in establishing the experimental 


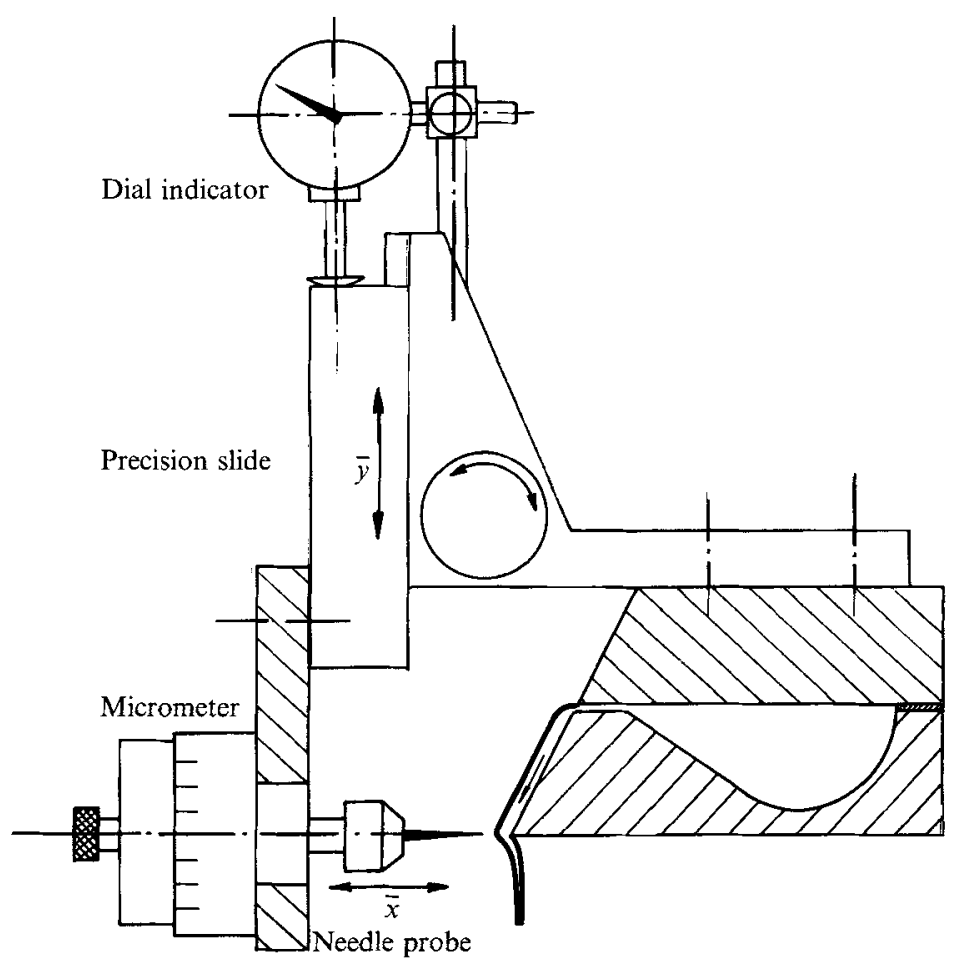

FIGURE 7. Mechanical micrometer needle probe.

$\bar{x}$ - and $\bar{y}$-positions at which the needle tip just contacted the sharp edge of the die. Other potential sources may have come from imprecisions in measuring the liquid properties and monitoring their temperature dependence, and from variations in flow rate over the width of the die.

A sheet of laser light was used to probe the teapot effect in two ways: it was used to section optically the sheet profiles in the sheet-forming zone; and it was used to measure the position of the static contact line where the curtain separated from the die surface.

Figure 8(a) shows the arrangement in the optical sectioning mode. The beam from a low-power laser (Model 1356P, Spectra Physics, San Jose, California, USA) was expanded into a sheet with a concave cylindrical lens $(90 \mathrm{~mm}$ focal length). To compensate for beam expansion normal to the sheet, a convex cylindrical lens $(300 \mathrm{~mm}$ focal length) focused the light into the zone of flow visualization. Small particles suspended in the test liquid scattered light when they resided in the illuminated plane. Both polystyrene latex particles of about $0.2 \mu \mathrm{m}$ (courtesy Professor W. R. Schowalter, Princeton University) and Mearlin Luster Pigment in the size range 15-40 $\mu \mathrm{m}$ (Mearl Co., New York, New York, USA) worked successfully. When recorded photographically with a camera oriented normal to the light plane, the streak lines of the particles generated two-dimensional images of the flow (see Figure 9 below).

To measure the location of the static separation line, the laser sheet was deflected from beneath toward the underside of the curtain die. Where the sheet met the steel surface, a straight line of refracted laser light appeared. That line was aligned with respect to a scale that was mounted on the underside of the die as shown in figure $8(b)$. The point where the line of refracted laser light intersected the contact line was very 
(a)

(a)
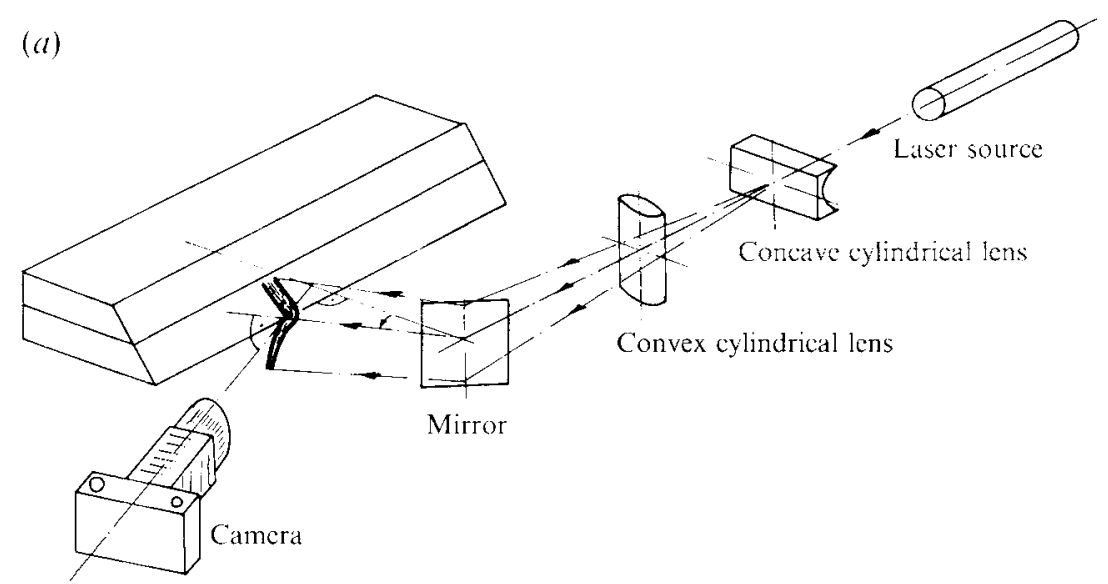

Mirror

(b)

$\mathfrak{l t}^{\prime}$

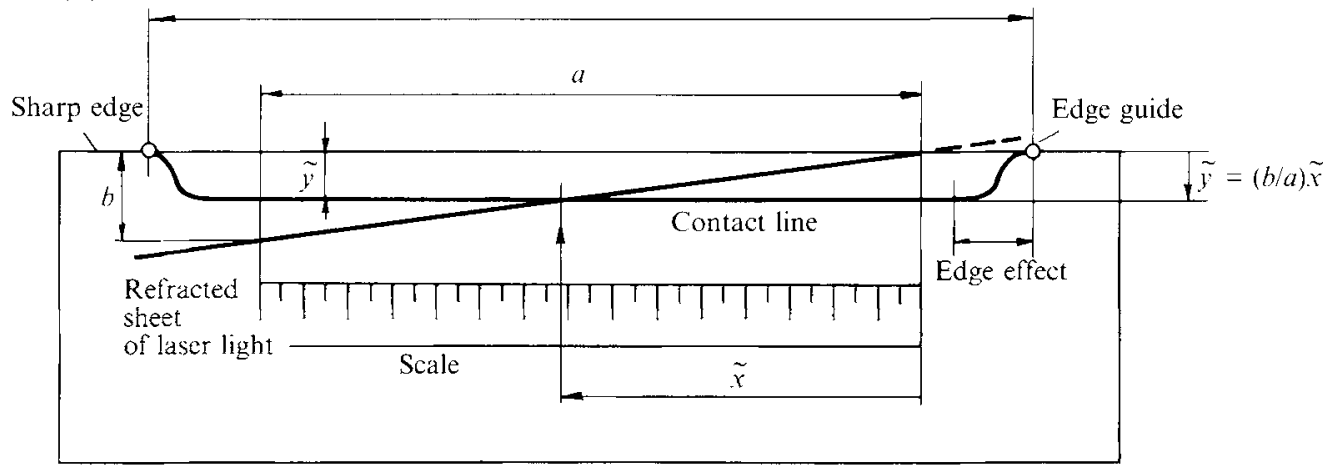

FIGURE 8. Laser light-sheet probe: $(a)$ optical sectioning set-up; $(b)$ set-up for measuring contact line position (view of die underside).

prominent. The measuring range $a$ was $150 \mathrm{~mm}$ and the distance $b 15 \mathrm{~mm}$. Such a small ratio $b / a$ makes the contactless measuring technique quite sensitive: with the resolution of $\tilde{x}$ taken to be $1 \mathrm{~mm}$, the contact line location could be determined to within $0.1 \mathrm{~mm}$. The shape of the contact line drawn in figure $8(b)$ is indicative of the contact lines that were observed in experiments when the liquid wet the lip underside. Edge effects induced by the edge guides were confined to a band at most $2 \mathrm{~cm}$ wide. Over a large central portion of the flow, the contact line was straight, and the curtain deflection was insensitive to the position at which it was measured.

\subsection{Experimental procedures}

The viscosity of the Newtonian glycerine-water mixtures ranged from 20 to $200 \mathrm{mPas}$, surface tension was typically around $0.063 \mathrm{~N} \mathrm{~m}^{-1}$, and density around $1210 \mathrm{~kg} \mathrm{~m}^{-3}$. Because the viscosity of the mixtures is highly sensitive to temperature and air humidity, it was monitored in every run. Surface tension and density were checked only sporadically, for they were found to be in good agreement with tabulated values (Newman 1968), so that measuring viscosity sufficed.

In experimental studies of the teapot effect, reproducible preparation of the solid surfaces is of foremost importance, because it can influence whether the liquid wets the 


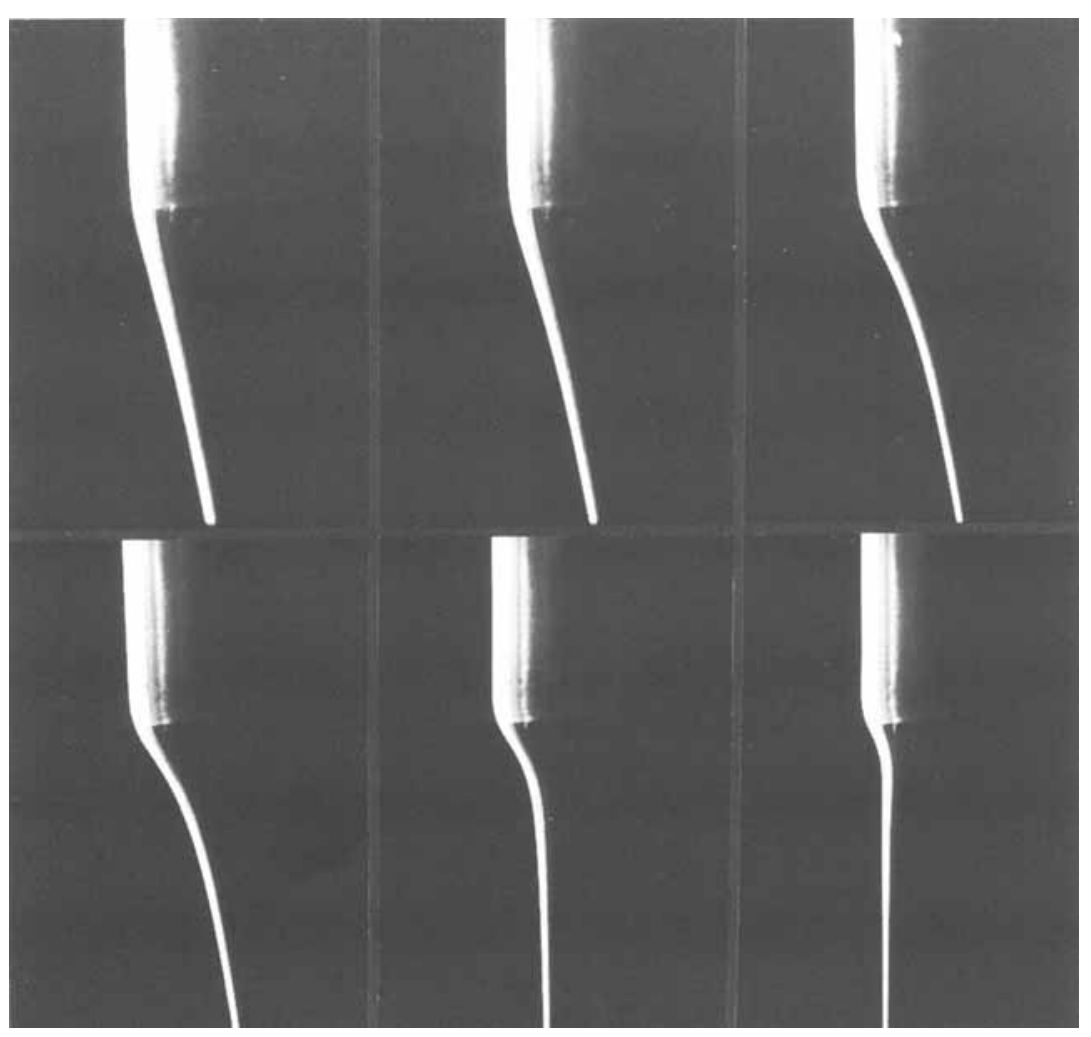

Figure 9. Hydrodynamic deflection of viscous liquid sheet falling from lip of vertical plane: section photographs.

underside of the lip. Between each run, the flow was stopped and the lip was washed with water, dried with a paper wiper ('Kimwipes' by Kimberly-Clark Corp., Roswell, GA), and cleaned with an acetone-soaked paper wiper. With this procedure, the measurements of contact line position were quite repeatable (see $\$ 6.2$ ) and, within the range of flow parameters reported here, the contact line appeared straight across a large central portion of the curtain as depicted schematically in figure $8(b)$. When flow parameters, in particular flow rate, were varied over wide ranges, many instability phenomena similar to those reported by Pritchard (1986) could be observed. The central experiments in the present study, however, focused on flow configurations most pertinent to curtain coating in which the liquid falls in the form of a stable sheet and separates from the solid surface at a straight contact line.

\section{Pinned contact line - purely hydrodynamic deflection}

\subsection{Effects of flow parameters on hydrodynamic deflection}

The trajectory of a liquid sheet that falls from the lip of a vertical plane changes substantially as flow rate varies. In the sequence of section photographs in figure 9 (taken as described in figure $8 a$ ), the flow rate decreases from top left to bottom right. At high flow rates, inertial effects dominate and so the liquid falls almost vertically. As the flow rate decreases, the liquid falls along a deflected, or 'antiballistic', trajectory. The deflection is most substantial at an intermediate flow rate. At low enough flow 
(a)

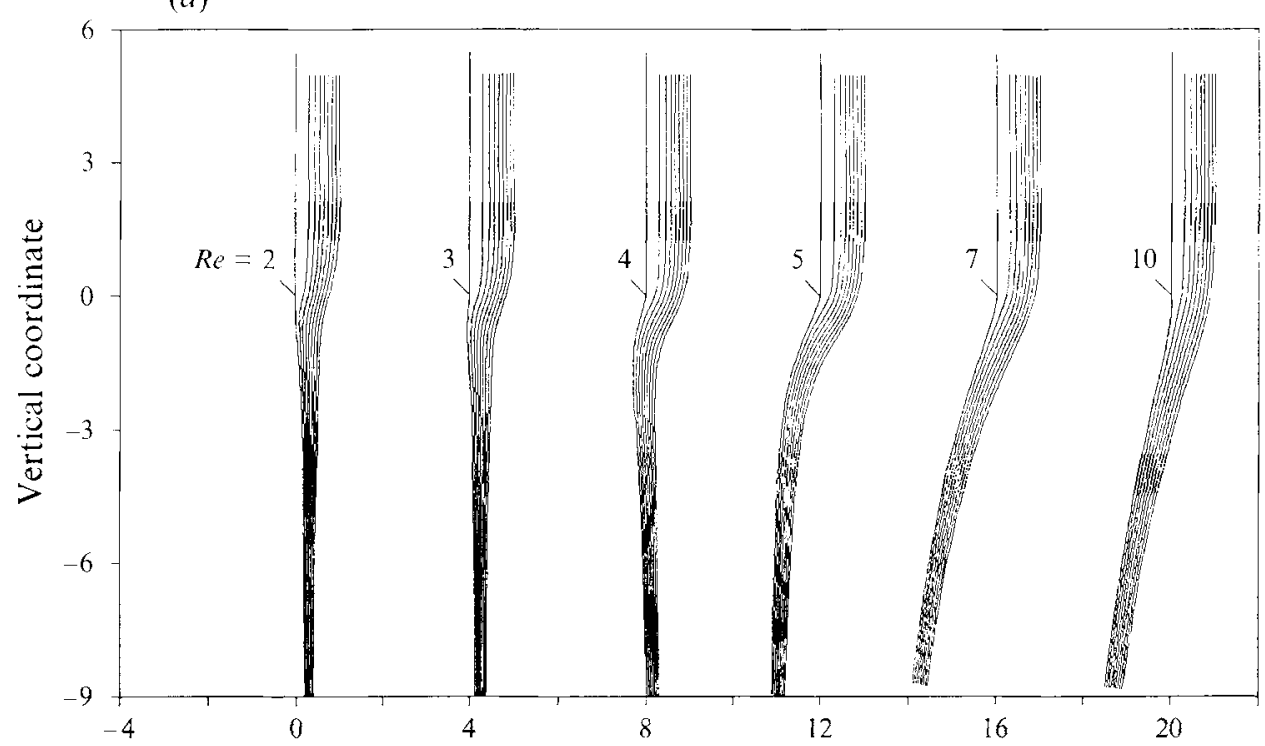

(b)

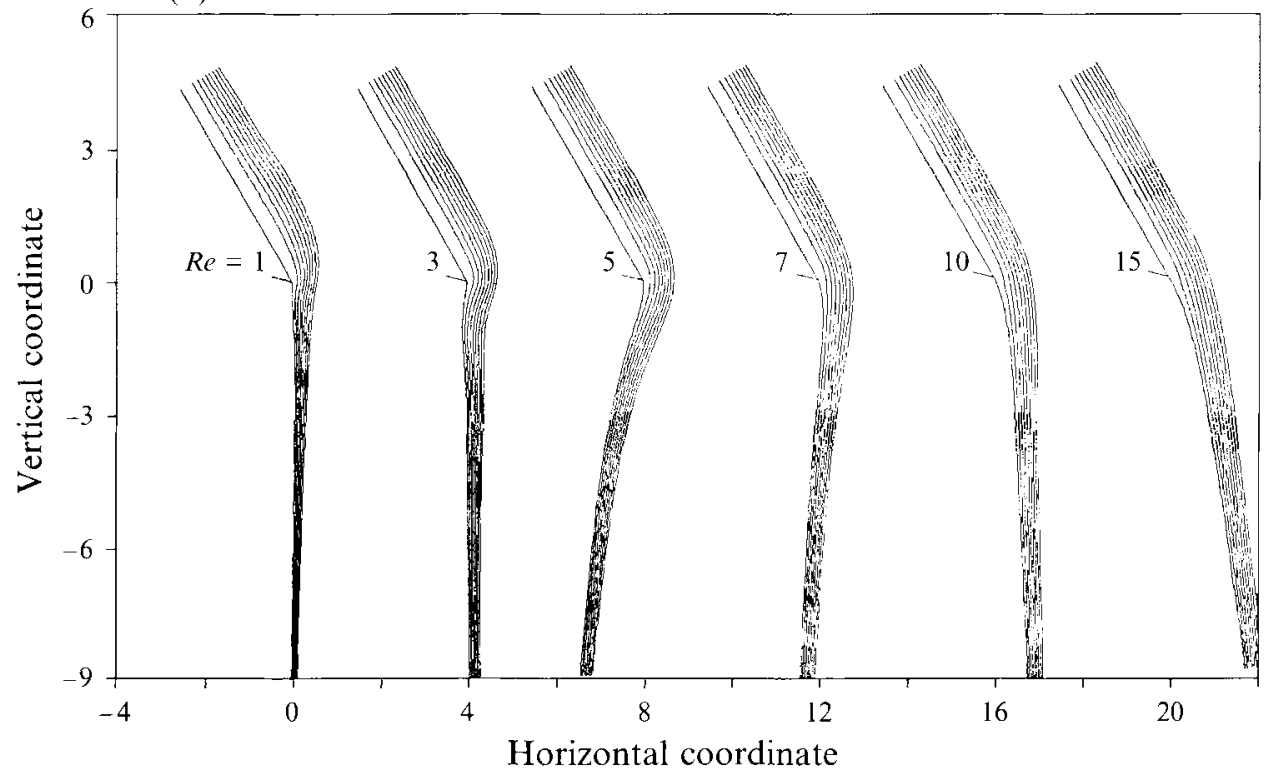

FIGURE 10. Hydrodynamic deflection of viscous liquid sheet falling from lip of vertical plane: computed profiles and streamlines for $(a)$ vertical plane $\left(\beta=90^{\circ}, N_{\sigma}=1.15\right)$; and $(b)$ inclined plane $\left(\beta=60^{\circ}, N_{\sigma}=1.0\right)$.

rates, viscous effects dominate and the flow rearrangement is confined to a short forming zone about the lip, so that the liquid curtain reaches vertical fall close to the lip.

In the flows depicted in figure 9 , the static contact line, when inspected by naked eye, seemed to be pinned at the sharp edge. Evidently, a viscous liquid sheet falling from the lip of an inclined plane deflects towards the underside of the lip because of the interplay of purely hydrodynamic forces, and not because of the submicroscopic forces that control wetting and spreading. 


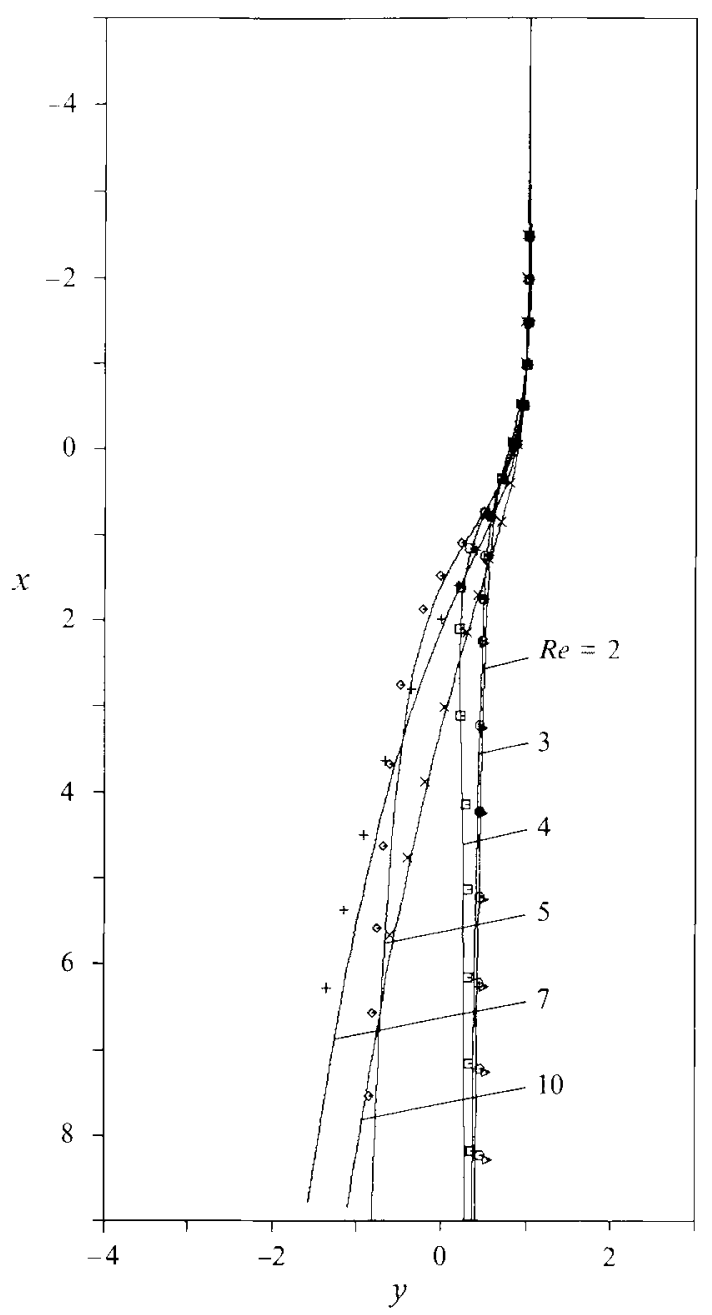

FIGURE 11. Comparison between predicted (solid lines) and measured (symbols) free-surface profiles $\left(N_{\sigma}=1.15, \beta=90^{\circ}\right)$.

Theory confirms that there is a purely hydrodynamic teapot effect, quite apart from wetting and spreading. Figure 10 shows sample predictions (computed as described in $\S 3.3$ ) of curtains that separate at the sharp edge as they fall from either a vertical or an inclined plane. The flow parameters in figure 10 are representative of the flow rates and physical properties of the glycerine/water mixtures used in the experiments of figure 9. For the case of a vertical plane, the theoretical predictions in figure $10(a)$ agree well with the section photographs in figure 9 . For the case of an inclined plane, the predictions of curtain fall in figure $10(b)$ reveal similar trends: the trajectory is 'ballistic' at high flow rates; reverses to an 'antiballistic' one as flow rate diminishes; and is nearly vertical at low rates.

The most striking characteristic of the hydrodynamic curtain deflection is that it is most pronounced at intermediate flow rates. Other features of the predicted flows worth noting include a thickening of the film just upstream of the lip at low flow rates (e.g. $R e=1$ in figure $10 b$ ) and a second inflexion point in some of the sheet trajectories (e.g. $R e=4$ in figure $10 a$, or $R e=3$ in figure $10 b$ ).

Free-surface profiles measured with the mechanical micrometer probe also agree 

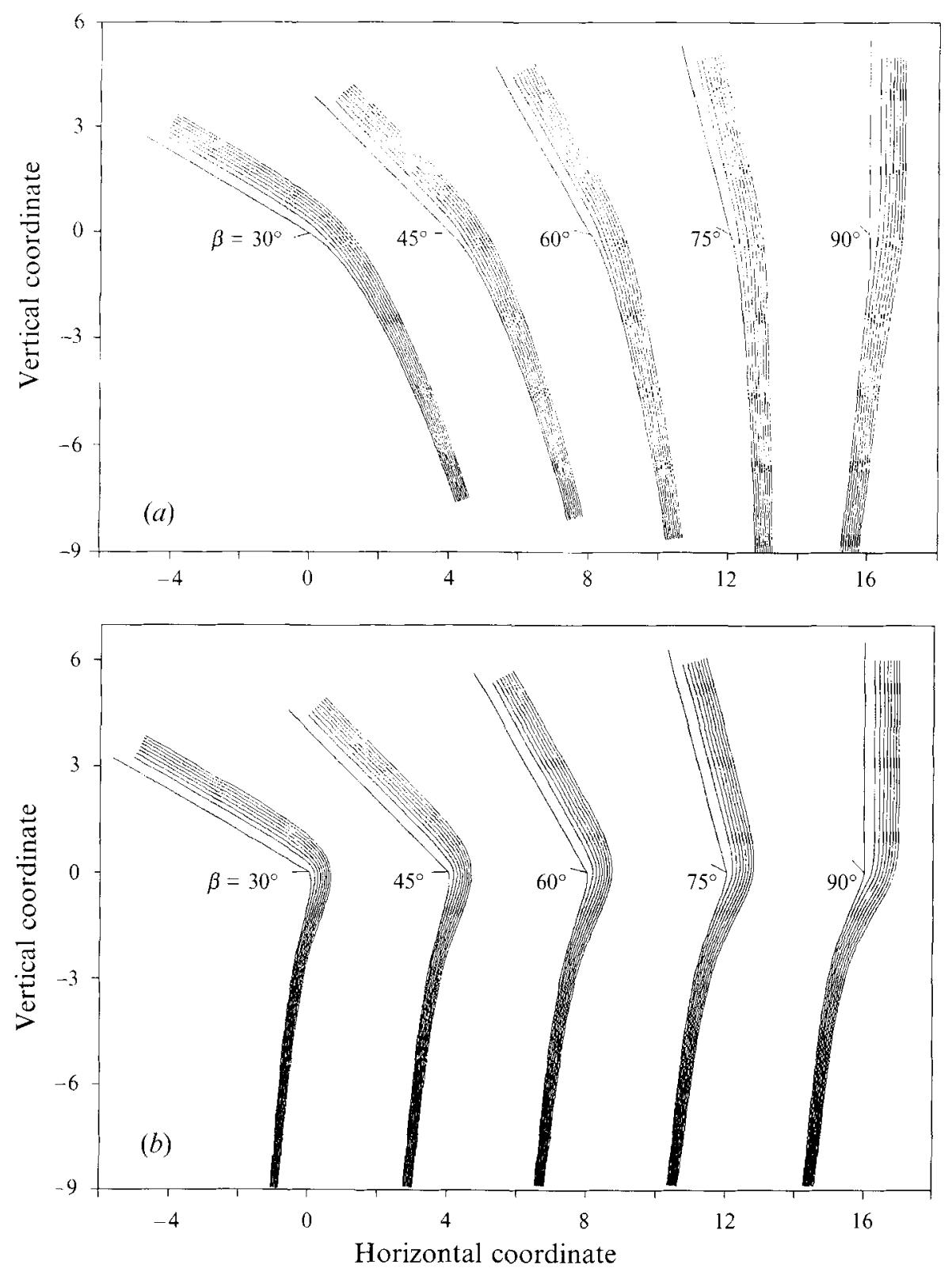

Figure 12. Effect of inclination angle $\beta$ on trajectory of viscous liquid curtain $\left(N_{\sigma}=1\right)$ : (a) high Reynolds number $(R e=20)$; $(b)$ intermediate Reynolds number $(R e=5)$.

closely with the theoretical predictions. In figure 11, the predicted top or outer free surfaces are drawn as solid lines, whereas the measured readings are marked by the various symbols. At the lower and higher Reynolds numbers, the agreement is excellent. Even such details as a second inflexion point in the free surface are the same in theory and experiment (see $R e=4$ ). At intermediate Reynolds numbers (namely $R e=5$ and 7), at which the deflection is greatest and most sensitive to flow rate, the agreement is not quite as good. The discrepancy between theory and experiment arises, most likely, from measurement errors of flow rate and viscosity, but may also stem from slight wetting of the lip underside (this is discussed further in $\S 6$ ). 

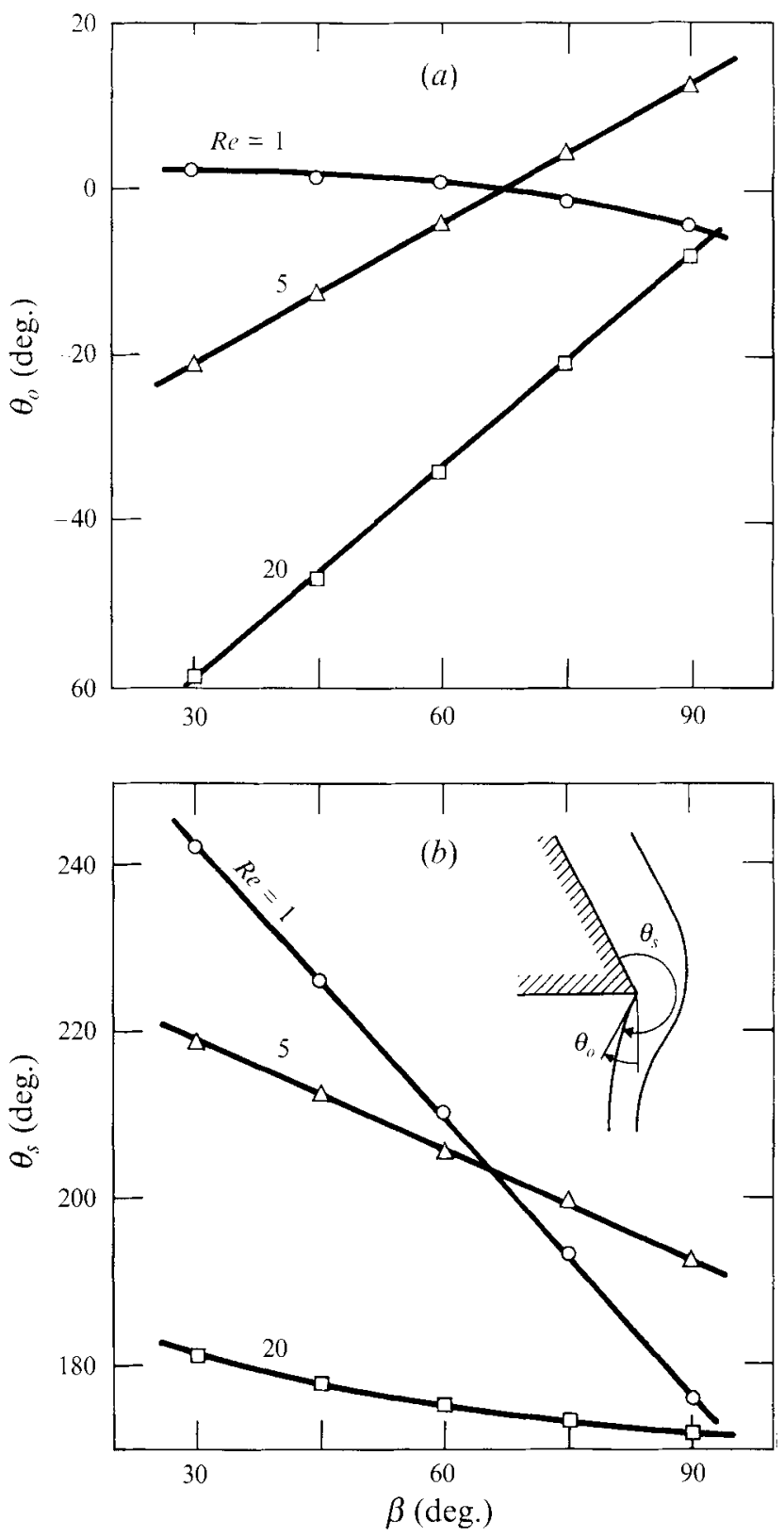

FIGURE 13. Hydrodynamic deflection of viscous liquid curtain $\left(N_{\sigma}=1\right)$ as a function of inclination angle $\beta$ : $(a)$ deflection angle $\theta_{0} ;(b)$ separation angle $\theta_{s}$.

As long as the contact line remains pinned at the sharp edge, the inclination angle $\beta$ is the only lip design parameter that influences the shape of the falling liquid sheet. At Reynolds numbers high enough that inertial effects dominate and the liquid falls along a ballistic trajectory, $\beta$ is all-important because it controls the initial slope of the sheet trajectory, as illustrated by the computed predictions in figure $12(a)$. In contrast, at intermediate Reynolds numbers at which the liquid follows an antiballistic trajectory, the angle $\beta$ influences the shape of the sheet surprisingly little, as shown in figure $12(b)$. Evidently, the purely hydrodynamic deflection is not connected with the 


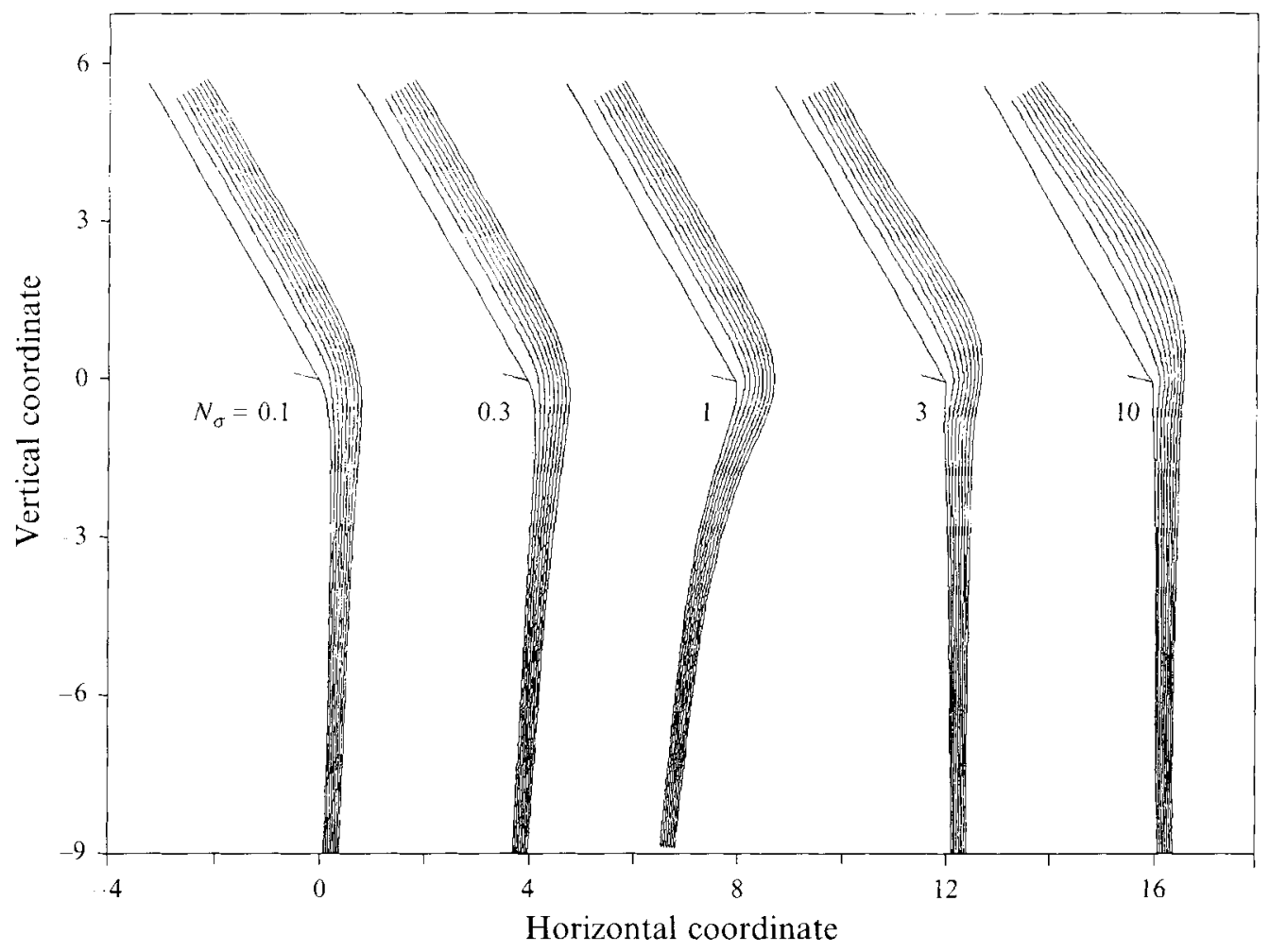

FIGURE 14. Hydrodynamic deflection of viscous liquid curtain: effect of capillary parameter $N_{\sigma} \equiv \sigma /\left[(4 \mu)^{4} g / \rho\right]^{\frac{1}{3}}\left(\operatorname{Re}=5, \beta=60^{\circ}\right)$.

inclination of the plane. At low enough Reynolds numbers that the liquid falls vertically just downstream of the lip (not shown in figure 12), the trajectory of the falling curtain changes even less as the inclination angle $\beta$ is varied.

These trends are summarized in figure 13(a) which shows the deviation from vertical of the rear free surface at the static attachment line, $\theta_{o}$, as a function of the angle of inclination of the lip, $\beta$. This deflection angle $\theta_{0}$ is most sensitive to $\beta$ at high Reynolds numbers, but remains almost the same at low Re. However, when and how the liquid wets the lip underside hinges not on the deflection angle $\theta_{0}$, but on the separation angle $\theta_{s} \equiv 270^{\circ}+\theta_{o}-\beta$ : to avoid wetting, $\theta_{s}$ ought to be as small as possible (see $\$ 6.1$ ). Figure 13(b) shows that $\theta_{s}$ is most sensitive to the inclination angle $\beta$ at low values of $R e$ for which $\theta_{0}$ scarcely changes, whereas at high Reynolds numbers changes in $\beta$ are nearly offset by accompanying variations in $\theta_{0}$ and thus, $\theta_{s}$ becomes insensitive to $\beta$. Figure 13 indicates that high Reynolds numbers are best to avoid the teapot effect and that, for any value of $R e$, the likelihood of wetting is smallest for large values of $\beta$.

Figure 14 shows how the purely hydrodynamic teapot effect is influenced by the property parameter, $N_{r}$, in which surface tension is paramount. The liquid curtain undergoes a significant deflection only at intermediate values of $N_{\sigma}$, whereas at low and high values the curtain falls vertically immediately downstream of the lip. The freesurface profiles in the latter two cases are, however, quite different: at $N_{\sigma} \gg 1$, there is a sizable standing wave upstream of the lip, whereas at $N_{\sigma} \ll 1$, there is virtually no upstream influence. Nonetheless, operating at a high or low value of the property parameter does not avoid the purely hydrodynamic teapot effect, as might be inferred from figure 14. Variations in $N_{\sigma}$ merely shift the range of Reynolds numbers $R e$, or flow 


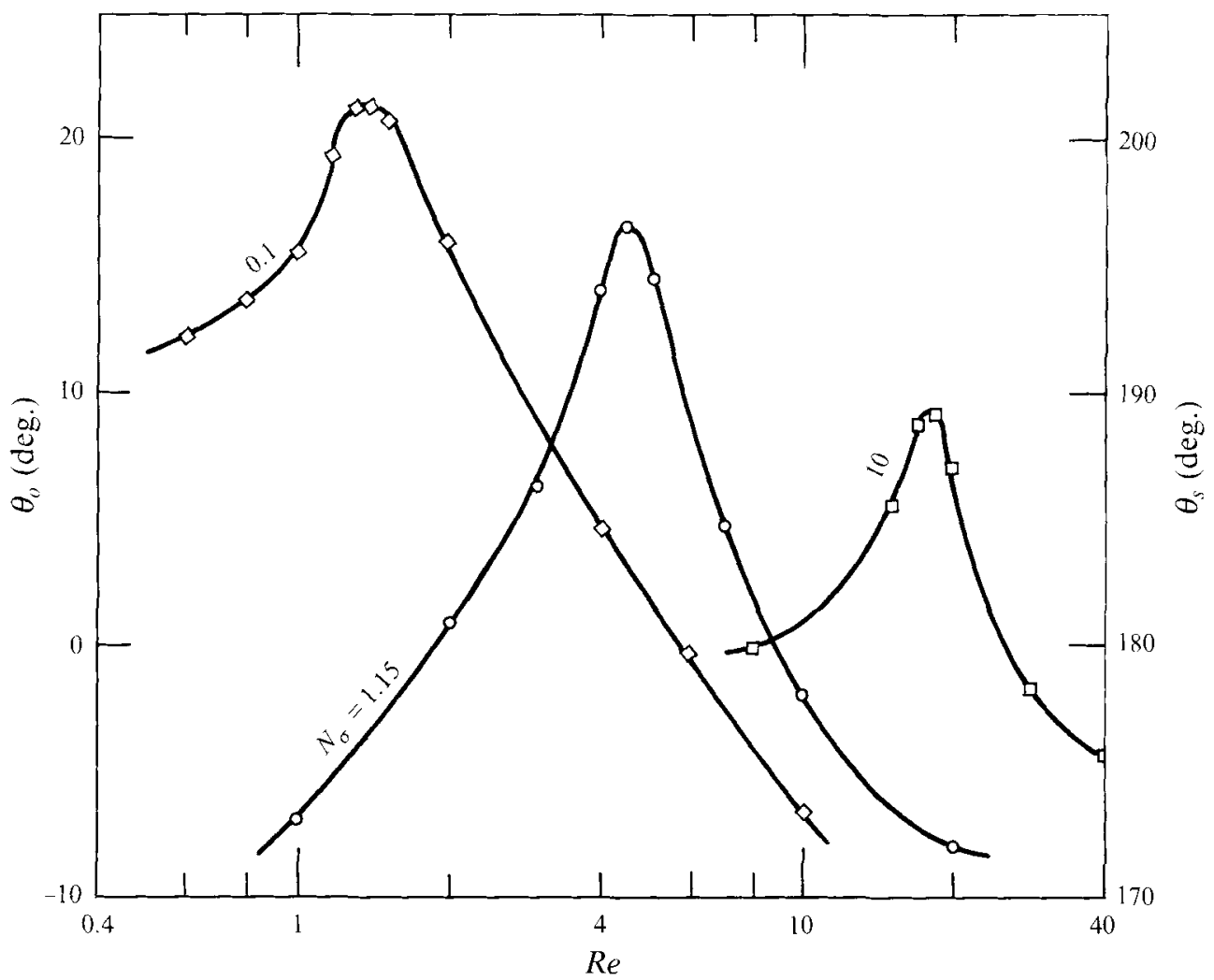

Figure 15. Summary of effect of flow parameters $R e$ and $N_{o}$ on hydrodynamic deflection of viscous liquid curtain $\left(\beta=90^{\circ}\right)$.

rate, in which the maximum deflection occurs, as is plain in figure 15. The maximum separation angle in each curve is not much different, even though $N_{\sigma}$ changes by orders of magnitude.

In summary, figure 15 captures the essence of the purely hydrodynamic teapot effect as far as it is controlled by flow rate and liquid properties: the curtain deflects toward the lip underside only in a certain range of flow rates which depends on viscosity and surface tension. This result clearly vitiates the conclusions Lin \& Krishna (1978) drew from their oversimplified macroscopic momentum balance. It also makes apparent the limited utility of potential flow calculations that ignore the effects of viscosity and surface tension altogether (Keller 1957; Vanden-Broeck \& Keller 1986).

\subsection{Nature of the hydrodynamic teapot effect}

The question remains of how to extract from the analysis a basic understanding of the teapot effect. Details of the computed flow fields in the forming zone and certain macroscopic balances of moment-of-momentum provide some insights, as follow.

The contours of kinematic and dynamic variables in figure 16 exemplify, at a set of flow parameters that yields a deflected curtain trajectory, the characteristics of flow rearrangement near the lip. The transition from shear to extensional flow is complex, yet the transition zone extends only a few film thicknesses upstream and downstream. Upstream, fully developed flow prevails as close to the sharp edge as 2 film thicknesses. The contours are those of a semi-parabolic velocity profile as given by (3), a linear hydrostatic pressure distribution, a linear variation of shear stress and vorticity in 


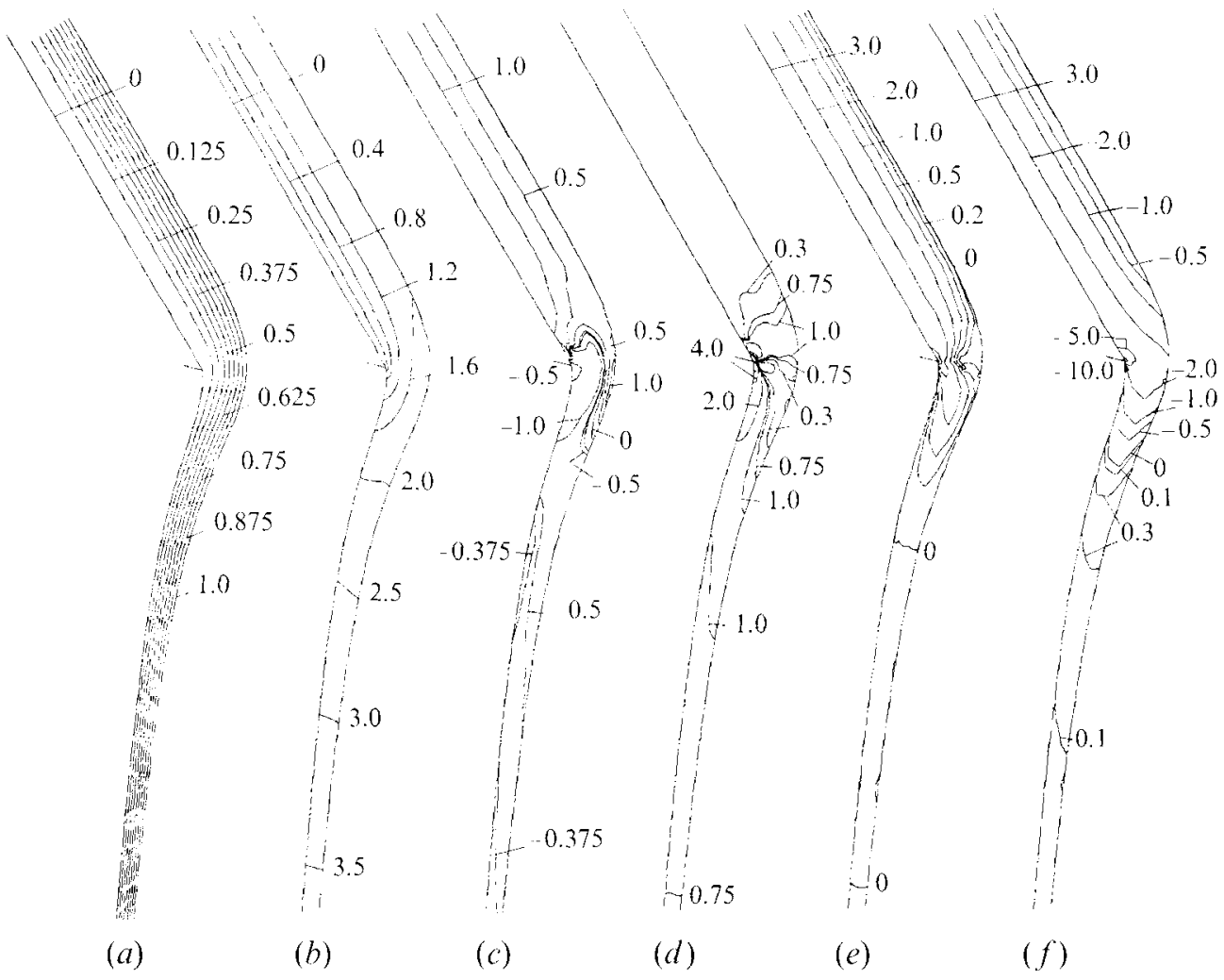

Figure 16. Details of flow field in sheet-forming flow near the lip of an inclined plane $(R e=5$, $\left.N_{r}=1.15, \beta=60^{\circ}\right):(a)$ stream function; $(b)$ particle speed $|v| ;(c)$ pressure $p ;(d)$ streamwise normal stress difference $\tau_{x *}-\tau_{n n} ;(e)$ shear stress $\tau_{n * x}$ normal to streamlines; $(f)$ vorticity $\omega_{z}$.

accord with (3), and a negligible normal stress difference. Downstream, nearextensional flow is established about 5 film thicknesses from the lip. The contours indicate a linear velocity profile that relaxes toward plug flow, the profile on which the approximate sheet profile equations (9) and (10) are based. The normal stress difference approaches a uniform profile, whereas pressure, which is subambient and approximately half the negative of the normal stress difference, varies linearly across the sheet, in agreement with the one-dimensional approximation (13). Shear stress is negligible, whereas vorticity, which here measures the local rate of rotation of the liquid as a rigid body, changes sign near the inflexion point of the sheet trajectory. In the immediate vicinity of the contact line, where the shear stress on the liquid surface suddenly disappears, the contours evidence the well-known singularity as predicted by a local analysis (Michael 1958), a rapid acceleration along the free surface, and a tensile normal stress which accompanies that acceleration.

The singularity at the wetting line and the complex flow nearby, together with the difficulty of locating the unknown free surfaces, argues against the possibility of fully explaining the teapot effect with any simple analytical approach. The situation is reminiscent of exit flow with die swell (cf. Richardson 1970; Silliman \& Scriven 1980), in which the swelling ratio is determined by the rearrangement of the flow field upstream of the exit plane. For that flow, a macroscopic balance that does not account for the modified velocity and stress distributions at the exit predicts a contraction of the viscous jet instead of swelling. 

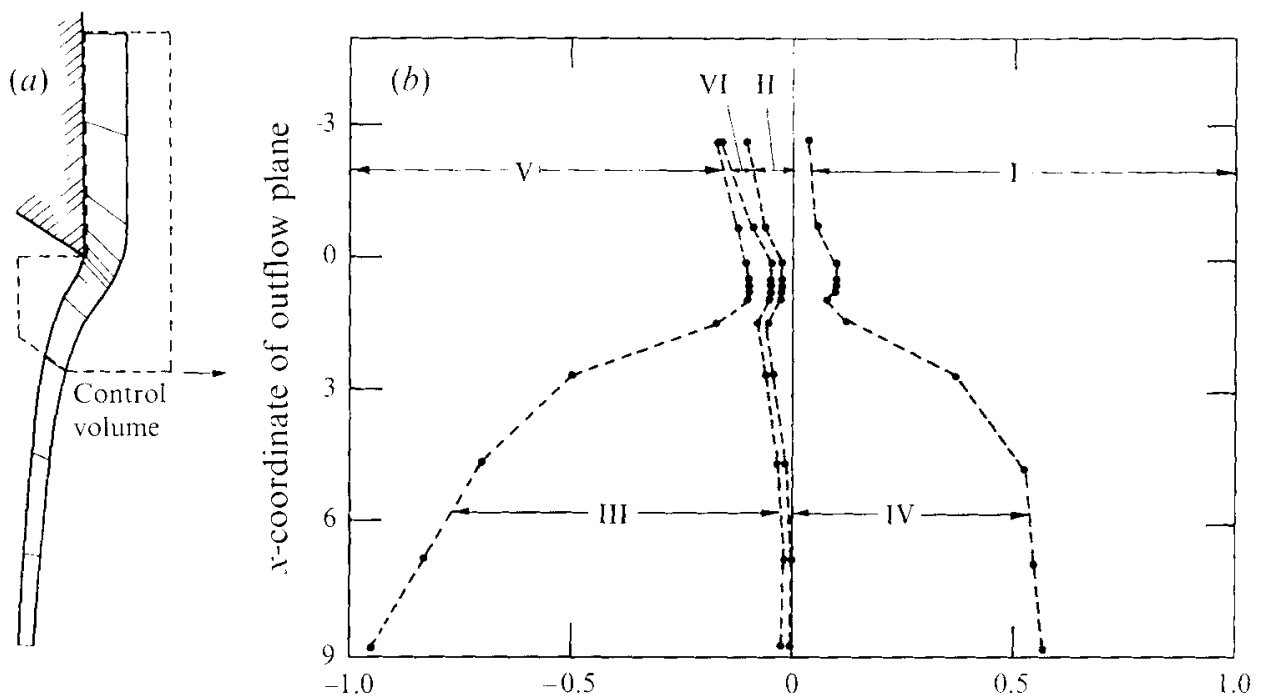

Normalized contributions to global moment-of-momentum balance

Figure 17. Macroscopic moment-of-momentum balance around viscous shcet-forming flow: $(a)$ definition of control volume: $(b)$ normalized contributions to (31) as a function of the r-position of the downstream boundary of control volume $\left(R e=5, N_{r}=1.15, \beta=90^{\circ}\right)$.

Nevertheless, the cause of the hydrodynamic teapot effect can be illuminated by means of a macroscopic balance of the torques exerted by the forces and momentum fluxes acting on a control volume of the sort shown in figure $17(a)$. With the origin at the sharp edge of the lip, that balance is
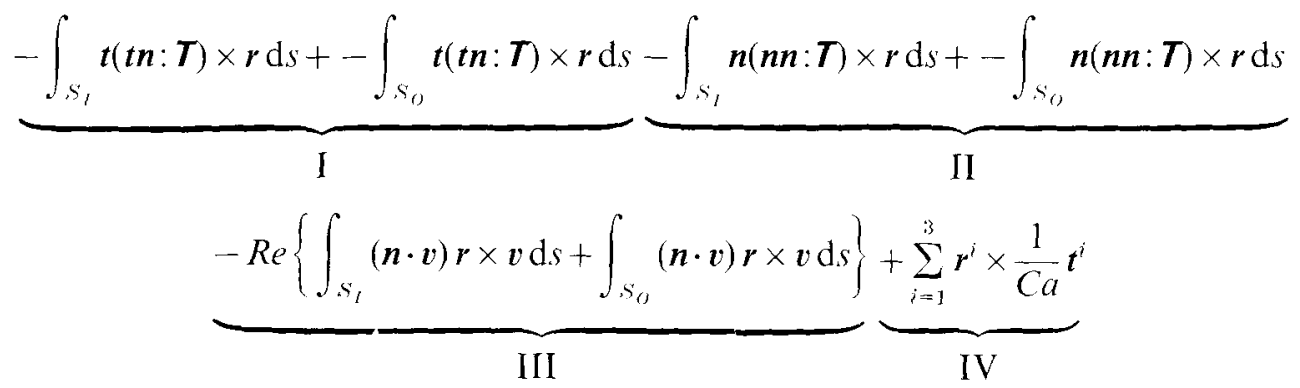

$$
\underbrace{+\frac{3}{\sin \beta} \int_{A} \boldsymbol{r} \times(\sin \beta \boldsymbol{i}-\cos \beta \boldsymbol{j}) \mathrm{d} A}_{\mathrm{V}}+\underbrace{\int_{S_{W}} \boldsymbol{n}(\boldsymbol{n} \boldsymbol{n}: \boldsymbol{T}) \times \boldsymbol{r} \mathrm{d} s}_{\mathrm{VI}}=0 .
$$

Locating the origin at the sharp edge brings the advantage that the wall shear exerts no torque. In (31), $S_{I}, S_{O}$ and $S_{W}$ denote the boundaries at the inflow plane, the outflow plane, and the slide surface respectively; $A$ is the area of the two-dimensional flow domain within the control volume; $\boldsymbol{t}$ and $\boldsymbol{n}$ denote the unit tangent and normal vectors to the respective boundaries; and $\boldsymbol{r}$ is the vector displacement from the origin. The term IV arises from the surface tension forces at the three locations where free surfaces intersect the control volume. The macroscopic balance (31) suggests that the relaxation toward plug flow and the absence of shear in the falling sheet are at the root of the hydrodynamic teapot effect. In fully developed film flow down a vertical wall, the 
moments of momentum inflow and outfiow and of surface tension forces cancel, whereas the transverse shear torques in the inflow and outflow planes are balanced by the torque of gravity. Were the curtain simply to fall vertically off the lip, with a straight rear free surface, and were the zone of flow relaxation short enough that gravitational acceleration were negligible, evaluating the terms in (31) would show that both the moment-of-momentum flow and the absence of shear torque downstream would cause an unbalanced torque that tended to deflect the curtain in the direction observed, and that surface tension would oppose the deflection.

Even though these insights are useful, it is impossible to predict the deflection accurately from a macroscopic balance alone. The results of the Galerkin/finiteelement analysis can, however, be used to compute the contributions to (31) in a postprocessing step, and thereby further clarify the source of the hydrodynamic teapot effect. Figure $17(b)$, in which the roman numerals refer to the terms in (31), shows how the various torques contributing to the macroscopic balance shift dramatically in their relative importance as the outflow plane of the control volume is moved in the vertical direction. Plainly, the hydrodynamic teapot effect results from a complicated interplay of the gravity torque $\mathrm{V}$, the viscous traction torques I and II, surface tension torque IV, normal traction torque VI at the wall, and moment-of-momentum inflow and outflow III. The flow parameters $R e$ and $N_{\sigma}$ reflect the physics behind the hydrodynamic deflection: as they change so do the relative significance of gravity, inertial, viscous and surface tension effects which contend with one another in a twodimensional flow; and so also do the development lengths of these effects upstream and downstream of the lip.

\section{Migrating contact line: wetting and hysteresis}

6.1. Inhibiting effect of a sharp corner on the spreading of a liquid curtain

Wettability of the lip underside can greatly influence the conditions under which steady states with a pinned contact line are stable with respect to contact line displacement away from the sharp edge. Figure 18 illustrates this in the case of curtain-forming flow from a vertical plane. The angle $\gamma$ between the two solid surfaces that form the sharp edge, defined in figure 1 and referred to as cut-back angle, is $60^{\circ}$ for this set of results. At a small contact angle $\theta_{c}$, the liquid wets the lip underside. Close to the lip, the main liquid stream deflects substantially, but does not climb in the direction against gravity. Instead, it drives a slowly circulating eddy in the corner-like region between the solid wall and the free surface. As $\theta_{c}$ is made larger, the contact line approaches the solid corner, the eddy shrinks, and the sheet trajectory resembles more and more that of a hydrodynamically deflected sheet with pinned contact line. Comparing, in figure 18 , the solution with pinned contact line with that at $\theta_{c}=60^{\circ}$ shows that slight wetting of the lip underside can readily account for the discrepancies between the computed and the experimental top free-surface profiles in figure 11. At a sufficiently high contact angle $\theta_{c}$, the contact line reaches the sharp edge, as is plain in figure 19 .

Figure 19 summarizes how the distance $x_{*}$ from the sharp corner of the solid to the contact line varies with contact angle. The filled triangle denotes the solution with pinned contact line on a type A tessellation (cf. figure 5); the open circles and squares distinguish two different finite-element tessellations, both of type B, with $N_{n}=5$ and $N_{n}=7$ respectively, $N_{n}$ being the number of elements across the sheet downstream of the lip. Evidently, the values of $x_{*}$ are insensitive to local refinement of the finiteelement discretization. As $x_{*}$ becomes small (namely $x_{*}<0.08$ ), continuation in contact angle $\theta_{c}$ leads to a constricted domain of convergence of Newton's method, presumably because of the proximity of the singularities at the contact line and the 


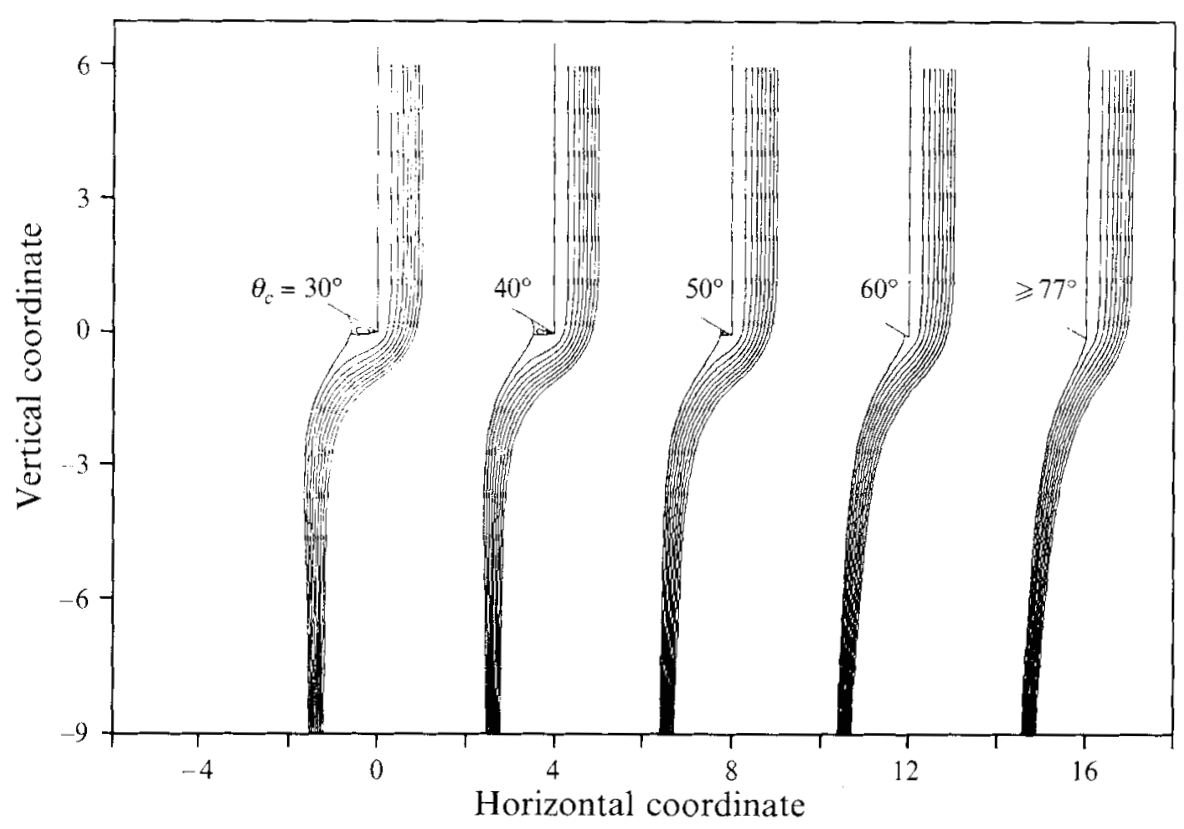

FIGURE 18. Effect of wettability on sheet-forming flow off the lip of a vertical plane $\left(\operatorname{Re}=5, N_{\sigma}=1.15, \beta=90^{\circ}, \gamma=60^{\circ}\right)$.

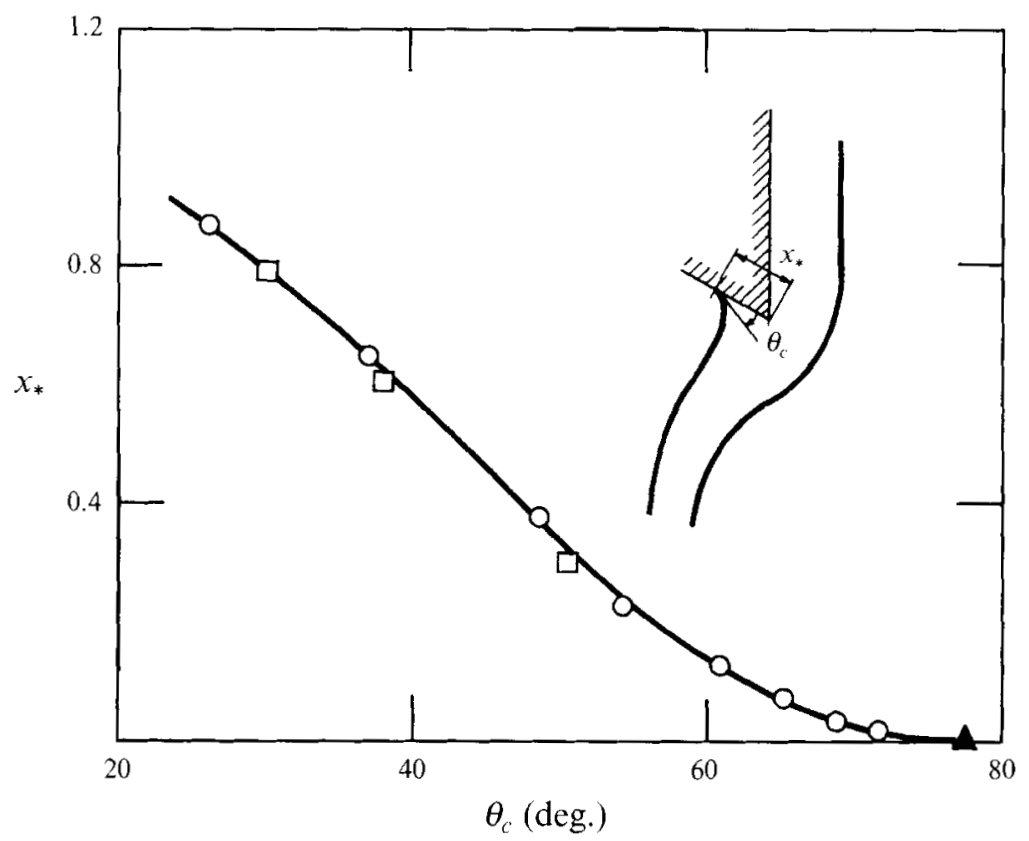

Figure 19. Inhibiting effect of sharp edge on wetting in sheet forming flow ( $R e=5, N_{\sigma}=1.15$, $\beta=90^{\circ}, \gamma=60^{\circ} ; \boldsymbol{\Delta}$, tessellation type A; $\mathrm{O}$, type B with $N_{n}=5 ; \square$, type B with $N_{n}=7$ ).

sharp edge; ultimately iteration does not converge at all. Continuation in contact line position $x_{*}$, however, avoids these convergence difficulties, and was used for $x_{*} \ll 1$.

Figure 19 conveys an important result about the inhibiting effect of a sharp edge on spreading of liquid: if the static contact angle $\theta_{c}$ is larger than a critical angle $\theta_{c}^{\text {crit }}$, there is always a steady-state configuration in which the lip underside is not wet by liquid. Figure 19 demonstrates that, as $x_{*}$ is set closer and closer to zero, the predicted contact 
angle $\theta_{r}$ approaches and eventually reaches $\theta_{r}^{r i t}=\theta_{*}-\left(180^{\circ}-\gamma\right)$, where $\theta_{s}$ is the separation angle that results when the contact line is taken to be pinned at the sharp corner. This finding implies that the contact line does not advance over the sharp edge if the separation angle $\theta_{s}$ satisfies the inequality condition

$$
\theta_{s}<\theta_{s}^{r i t}=\theta_{c}+\left(180^{\circ}-\gamma\right)
$$

In the teapot flow analysed here, $\theta_{s}$ is a function of the flow parameters $R e$ and $N_{\sigma}$ and of the inclination angle $\beta$ (cf. $\$ 5$ ), whereas $\theta_{*}^{c r i t}$ depends on the contact angle $\theta_{r}$, which here must be a recently advanced angle as discussed further below, and on the cut-back angle $\%$

Condition (32) is, of course, the familiar Gibbs inequality condition which Gibbs (1906) derived from a purely geometric extension of the familiar Young-Dupré equation. It has been confirmed experimentally for the equilibrium of a static drop bounded by the sharp edge of a solid disk (Oliver et al. 1977). The results in figures 18 and 19 argue that the inequality (32) applies equally well to dynamic situations in which flowing liquid separates from a rigid wall at a sharp edge. Apparently, the inhibiting effect of a sharp edge on liquid spreading arises merely from a geometrical constraint in flow situations as well. Further experimental confirmation of this finding is desirable, however.

A real edge is not truly sharp, of course. Nonetheless, what appears to be an edge effect is normally observed at the macroscopic level. If an edge is modelled as a round surface without discontinuities, the contact line movement that is needed to maintain a constant contact angle becomes smaller as the radius $R_{e}$ of the edge decreases; as $R_{e}$ approaches zero, the apparent contact line seems pinned (cf. Oliver et al. 1977).

\subsection{Hydrodynamic hysteresis}

The Gibbs inequality condition (32) is a necessary, but not a sufficient, condition for the contact line not to take a position beyond the sharp edge. It admits combinations of $\theta_{c}$ and $\gamma$ at which computed predictions with the contact line pinned at the edge correspond to experimentally realizable flow states. However, at a particular set of flow parameters at which $\theta_{s}<\theta_{s}^{c r i t}$, there might be other steady-state configurations in which the liquid wets the lip underside.

An example of such multiple steady states is shown in figure 20 . Even though the cutback angle $\gamma=60^{\circ}$ is the same as in figure 18 , the contact line position $x_{*}$ depends on the contact angle $\theta_{c}$ in a strikingly different way, primarily because the plane is inclined at $60^{\circ}$ so that the lip underside becomes horizontal. When the liquid wets the solid sufficiently well (in this particular case for $\theta_{c}<78^{\circ}$ ), the only realizable position of the static separation line is one a few film thicknesses back from the lip. On the other hand, when the liquid wets the solid poorly (here for $\theta_{c}>97^{\circ}$ ), the only steady-state flow is that with a pinned separation line. For intermediate contact angles $78^{\circ}<\theta_{c}<97^{\circ}$, however, multiple steady states exist, and these give rise to hysteresis. The solution family in figure 20 forms an ordinary hysteresis loop with two turning points. These mark loss of existence of steady-state solutions and signal an abrupt change in the structure of the flow when a parameter, here the contact angle $\theta_{c}$, is altered by a small amount beyond its value at the turning point. At the point where the family of solutions with a freely locatable separation line branches off the abscissa, which represents solutions with a pinned contact line, the Gibbs inequality condition (32) applies as in figure 19.

The computed hysteresis loops are quite insensitive to further refinement of the finite-element tessellation. Figure 20 , for instance, indicates that even the location of 


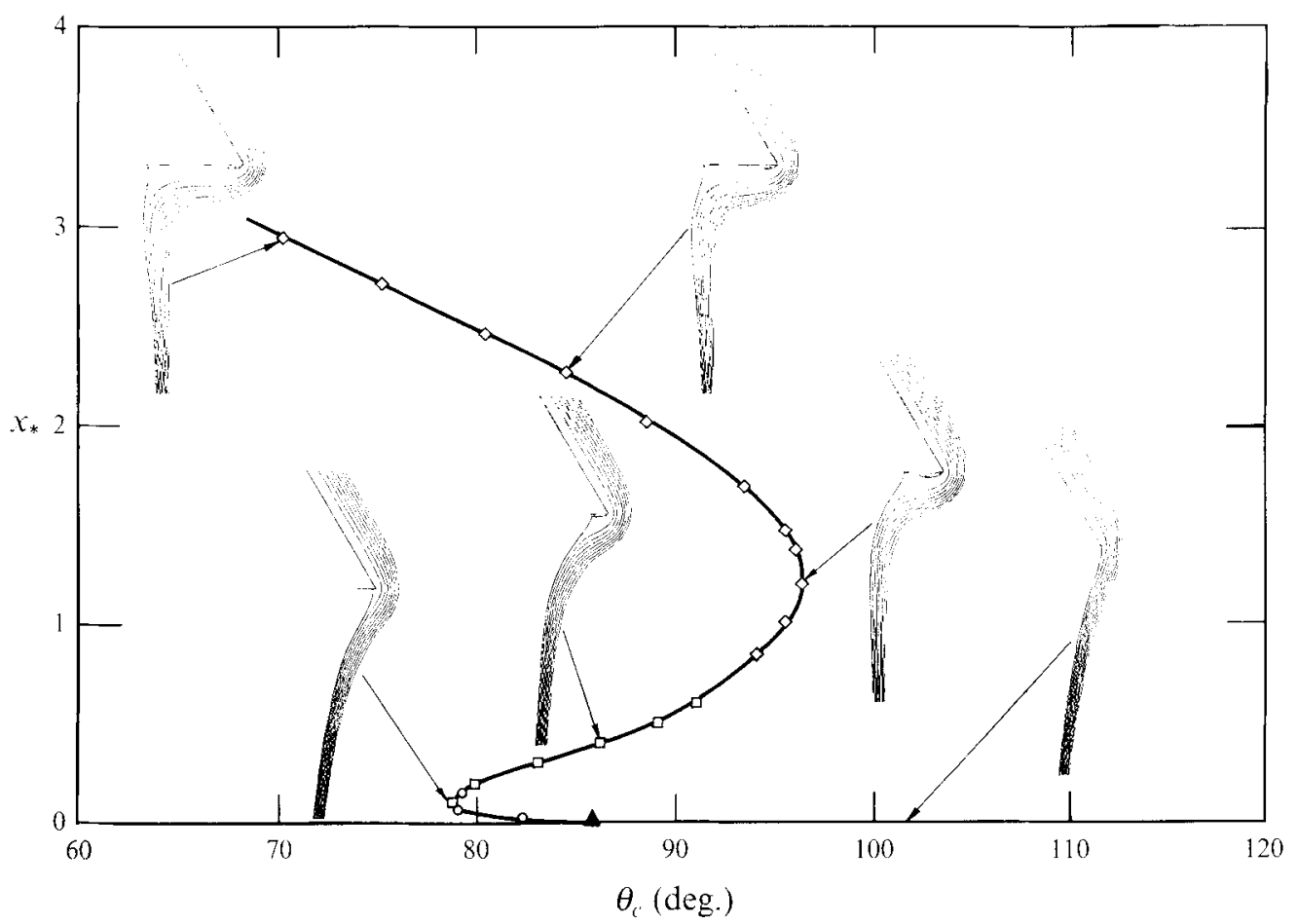

Figurf. 20. Hysteresis loop for contact line position $x_{*}$ as a function of contact angle $\theta_{c}$ ( $R e=5$, $N_{n}=1, \beta=60^{\circ}, \gamma=60^{\circ} ; \diamond$, tessellation type C; $\square$, type B with $N_{n}=7 ; \bigcirc$, type B with $N_{n}=5$; $\Delta$, type A).

the lower turning point, where the solution family curves sharply, is virtually unaffected when $N_{n}$ in tessellation B is changed from 5 (circles) to 7 (squares).

Stability of a particular flow state can be inferred from its location in the tree structure of families of steady states (e.g. Iooss \& Joseph 1980). The sheet-forming flows analysed here must change stability at a turning point in either $\theta_{e}$, as in figure 20 , or any other parameter $\Pi_{j}$, as illustrated below. Stability deduced from the tree structure is, of course, only with respect to a restricted class of disturbances, namely two-dimensional non-oscillatory ones. In the light of other situations with similar hysteresis loops, it seems most likely that the upper and lower branches are stable, whereas the branch that connects the two turning points is unstable. Experiments reported below establish that the upper branch of the hysteresis loop and the branch on the abscissa indeed correspond to stable flow states, so that the branch between the turning points must be unstable. Incidentally, in the states on the branch, a sizeable eddy separates the main flow completely from the lip underside (this detail in the flow field is most easily seen in the solution at $R e=7$ in figure 22 below).

The change in stability at the turning points can be confirmed in the computations by monitoring the determinant of the Jacobian matrix $\boldsymbol{J}$ of the Newton iteration for each converged solution, because $\operatorname{det}|\boldsymbol{J}|$ is just the product of the eigenvalues of that matrix. Figure 21 confirms that at each turning point the sign of $\operatorname{det}|\boldsymbol{J}|$ does indeed change, corresponding to an odd number of eigenvalues passing through zero $(\boldsymbol{J}$ is constructed here for the case of a prescribed contact angle $\theta_{c}$ and free contact line position $x_{*}$ ).

Compared to wettability of solid surfaces, flow rate is more readily varied in 


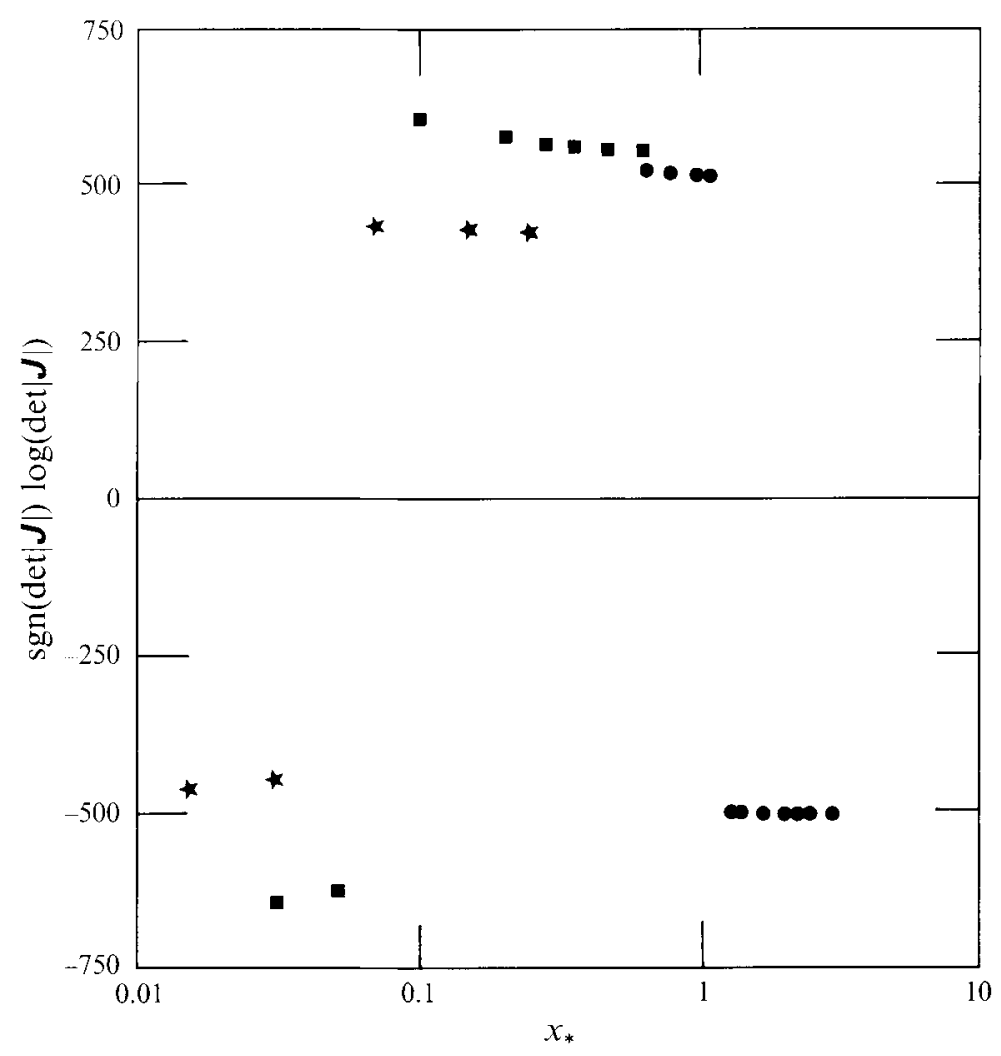

FiguRE 21. Change in sign of determinant $\operatorname{det}|\boldsymbol{J}|$ of Jacobian matrix of Newton's method in the vicinity of turning points for the solution family displayed in figure 20 ( $R e=5, N_{\sigma}=1, \beta=60^{\circ}$, $\gamma=60^{\circ}$

, tessellation type $\mathrm{C}$

ם, type B with $N_{n}=7 ; \star$, type B with $N_{n}=5$ ).

experiments, and more relevant to practical curtain coating. Figure 22 reveals a hysteresis loop in contact line position $x_{*}$ as a function of Reynolds number $R e$ at a fixed contact angle $\theta_{c}=67^{\circ}$. This loop accords with that in figure 20, for the lip configuration is the same, and so figures 20 and 22 correspond to planes of constant $\theta_{c}$ and $R e$ in the $\left(x_{*}, R e, \theta_{c}\right)$-space, the curves being intersections of those planes with a surface, or manifold, of states at fixed values of $N_{r}, \beta$ and $\gamma$.

At low Reynolds numbers, the only steady states in figure 22 are those in which the underside of the lip is wet and the curtain falls almost vertically. When the sheetforming flow is started up at a low flow rate, this configuration ought to be established. As the rate is increased, the contact line advances, the deflection of the curtain intensifies, and the liquid stream detaching from the solid beneath the sharp edge drives a recirculating eddy of increasing size. At $R e_{U}$, the eddy has grown so big that the main flow does not reattach at all to the lip underside, and the upper turning point is reached. At Reynolds numbers beyond $R e_{U}$, the Navier-Stokes system has no steady two-dimensional solutions with a free contact line. Thus a slight increase of flow rate beyond the critical value $q_{U}=R e_{U} \mu / \rho$ causes a sudden flow transition: the separation line recedes spontaneously to the sharp edge, as indicated by the broken arrow in figure 22. As flow rate is increased further, the curtain-forming flow behaves as described in $\S 5$.

When, on the other hand, the flow is started up at a high enough rate that $R e>R e_{U}$, the contact line remains pinned, or moves imperceptibly, as flow rate is decreased until 


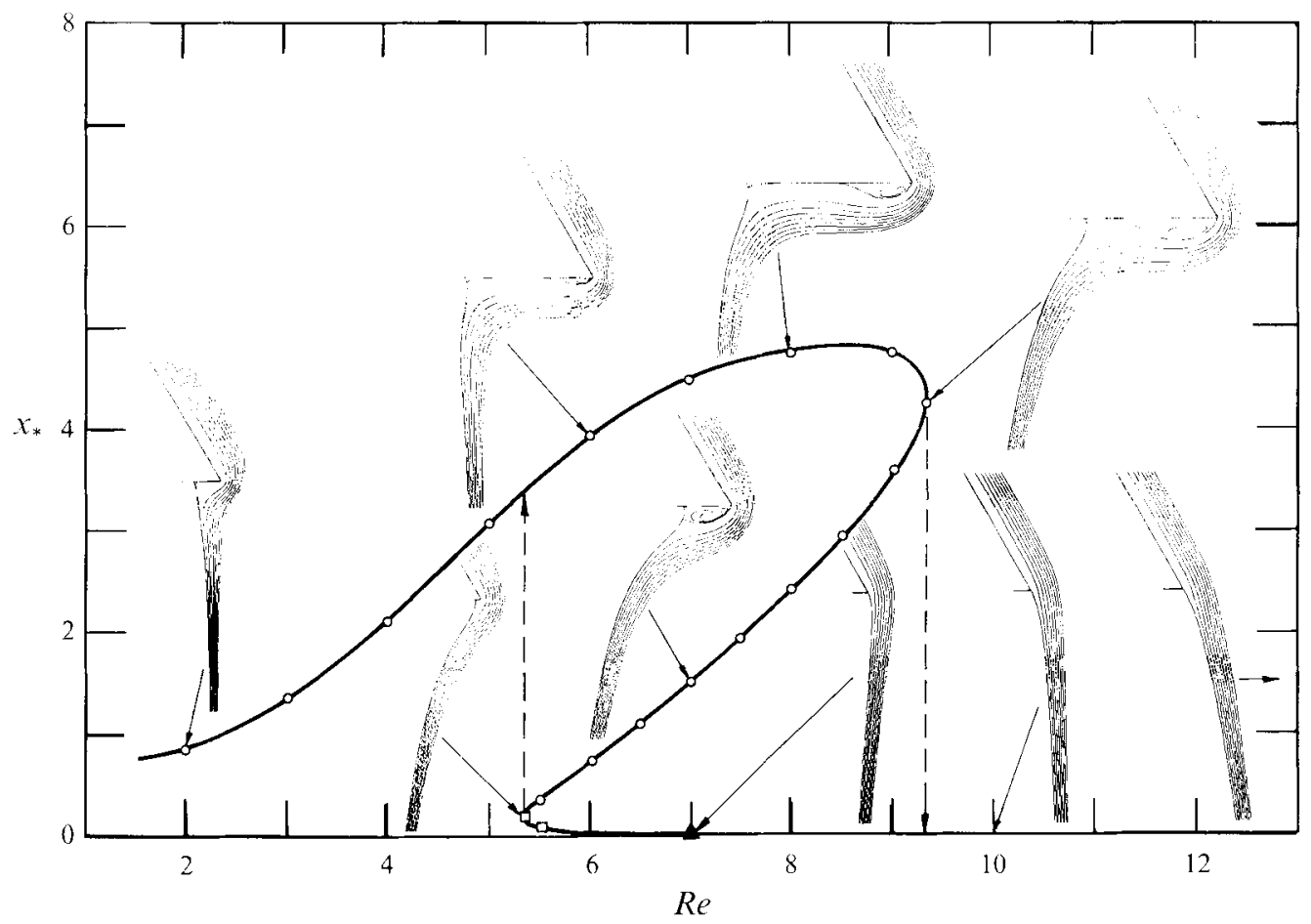

Figure 22. Hydrodynamic hysteresis for contact line position $x_{*}$ as a function of Reynolds number, or flow rate $\left(N_{\sigma}=1, \theta_{c}=67^{\circ}, \beta=60^{\circ}, \gamma=60^{\circ} ; \bigcirc\right.$, tessellation type C; $\square$, type B; $\boldsymbol{\Delta}$, type A).

the Reynolds number reaches a critical value $R e_{s}$, at which the separation angle $\theta_{s}$ no longer satisfies the Gibbs inequality condition (32). Decreasing Re further makes the contact line detach from the sharp edge. The line remains, however, close to the edge down to a flow rate that corresponds to a critical value $R e_{L}$ at the lower turning point. A further decrease in flow rate triggers another sudden transition that re-establishes the flow configuration on the upper branch with a substantial portion of the lip underside being wet, and a large recirculating eddy.

The multiple steady-state solutions in figure 22 give rise to a hysteresis effect that originates solely in the competition of hydrodynamic forces which interact in a nonlinear manner. The difference between co-existing flow configurations can be quite dramatic, with obvious practical implications for start-up of coating operations.

The theoretically predicted hydrodynamic hysteresis has been verified in the laboratory. Figure 23 summarizes three sets of measurements of wetting line position by the laser-sheet refraction technique explained at figure $8(b)$. The photographs complementing the data were taken with the laser-sheet sectioning technique described at figure $8(a)$. The ordinate in figure 23 is the flow-rate independent dimensionless position $x_{*} R e^{\frac{1}{3}}=X_{*}\left[\rho^{2} g \sin \beta /\left(3 \mu^{2}\right)\right]^{\frac{1}{3}}$, where $X_{*}$ is dimensional. Each data point is the average of seven separate runs. The time interval between incrementally increasing or decreasing flow rate was at least 5 minutes. This was found to be long enough for the new flow state to become established. The vertical bars represent the standard deviation in the experimental data.

In data set 1 (closed squares) the flow was started up at a low pumping rate and raised in increments. The curtain shapes in the photographs and the curve of measured contact line positions closely resemble the theoretical predictions in figure 22 . In the 


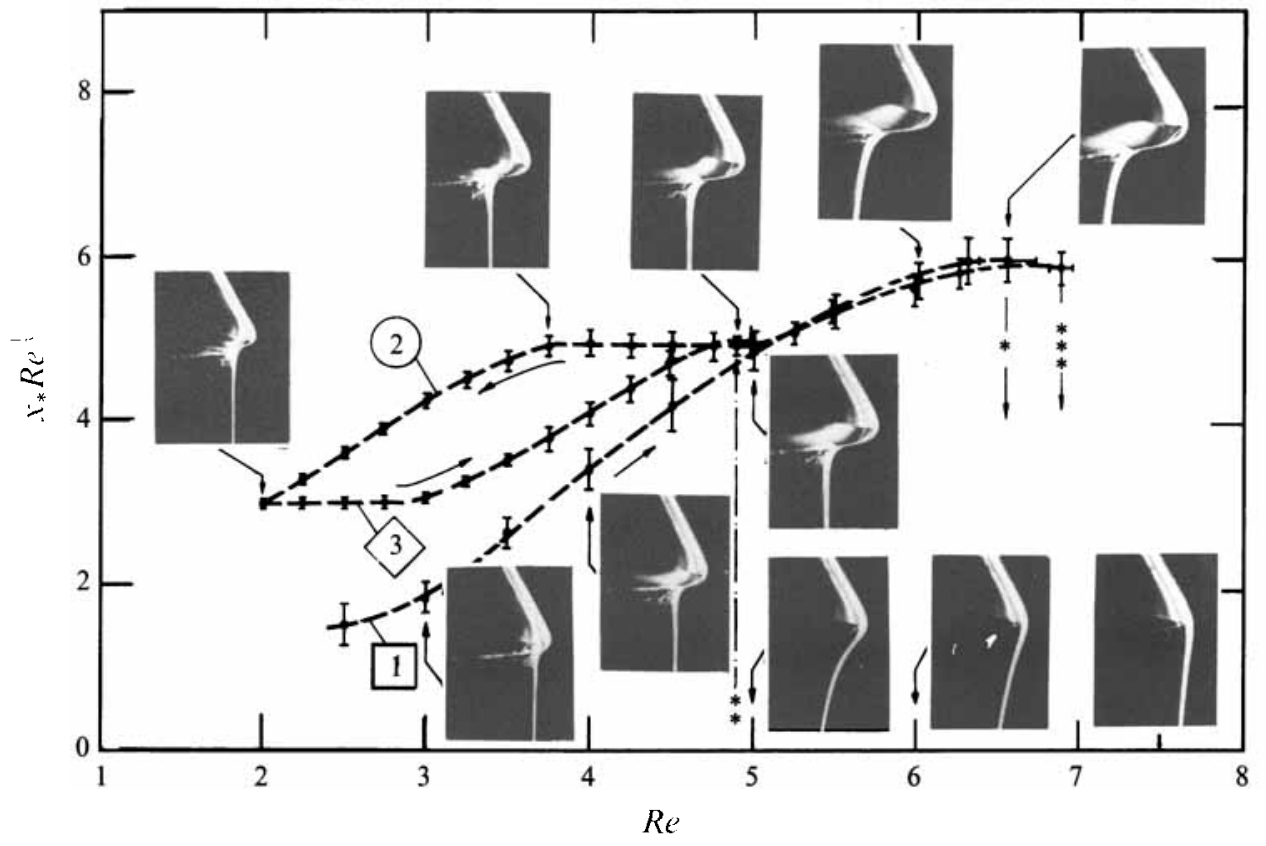

FIGURE 23. Experimental evidence of hydrodynamic hysteresis and contact-angle hysteresis $\left(N_{\sigma} \approx 0.8, \beta=60^{\circ}, \gamma=60^{\circ}\right.$; the symbols are explained in the text).

experiment, too, there was an abrupt flow transition: at an upper critical Reynolds number indicated by an asterisk, a minute rise in flow rate triggered retraction of the contact line to the sharp edge. Retraction commenced near the two edge guides and propagated toward the centre of the curtain at a speed of a few millimetres per second. Eventually, the curtain separated from the lip at the sharp edge over its entire width, even though patches of liquid were occasionally left behind on the lip underside. As pumping rate was increased further, the flow behaved as described in $\S 5$.

In data set 2 (solid circles) the flow was started up at $R e>R e_{U}$, i.e. above the upper turning point of figure 22 . The contact line assumed a pinned position at the lip, and remained pinned as flow rate was lowered in increments down to values at which there was substantial wetting in the previous case. Thus, the experiments confirm that there can be remarkably different flow configurations at the same flow rate (e.g. $R e=5$ ). The slight wetting of the lip underside which the theory predicts at $R e_{L}<R e<R e_{S}$ was difficult to confirm in the experiment. At a lower critical value of flow rate, indicated by two asterisks in figure 23 , the contact line was observed to jump back spontaneously, starting in the middle of the curtain and spreading toward the edge guides at a speed of a few millimetres per second. Eventually the contact line assumed a shape like that depicted schematically in figure $8(b)$, where the contact line is straight over most of the die width, with edge effects being confined to the vicinity of the edge guides. As the flow rate was lowered further, the contact line remained stationary over an appreciable range, but at lower rates took up a progression of positions closer and closer to the sharp edge of the lip.

In data set 3 (solid diamonds) in figure 23 , the flow rate was reversed just above the minimum needed to maintain a stable curtain $\left(\sim 3 \times 10^{-3} \mathrm{~m}^{3} \mathrm{~s}^{-1}\right)$. Upon raising the rate in increments, the contact line at first remained stationary; then took up successively advanced positions along a curve close to that of set 1 ; remained 
stationary over a small range of flow rates close to $R e_{3}$; advanced along a curve virtually identical to that of set 1 ; and finally retracted to the sharp corner of the lip at an upper transition point, indicated by three asterisks, in similar fashion to set 1 .

\subsection{Contact-angle histeresis}

Together, the data sets 2 and 3 in figure 23 form another hysteresis loop, which is quite distinct from that of the hydrodynamic hysteresis in figure 22, but which strongly resembles that of the contact-angle hysteresis, well known from many slowly equilibrating systems (cf. Teletzke et al. 1987, 1988): the contact line advances from position to position as flow rate is incrementally raised, in order to maintain an apparent contact angle characteristic of the solid-liquid-gas combination when the liquid displaces gas; and the contact line retracts reluctantly, as flow rate is incrementally lowered, in order to maintain an apparent contact angle that is lower and characteristic of situations where liquid has recently been withdrawn from a solid surface. This kind of hysteresis has been predicted, for instance, for a drop resting on a surface whose roughness consists of concentric grooves (Huh \& Mason 1977), and it has been measured on similar surfaces (Oliver et al. 1980). Force-versus-depth curves for plates being immersed and withdrawn from liquid exhibit analogous hysteresis loops (Johnson \& Dettre 1969).

Theoretical predictions of contact line position confirm that the hysteresis loop drawn in figure 23 can indeed be attributed to the differences between a recently advanced apparent contact angle $\theta_{c}^{a}$ and a recently receded one $\theta_{c}^{r}$, the timescale since the last change being that mentioned above, i.e. about five minutes. In figure 24 , theoretical curves of $x_{*} R e^{\frac{1}{3}} v s$. Re are superimposed on the experimental data of figure 23. The contact angles in the computations are chosen so that the theoretical value of $x_{*} R e^{\frac{1}{3}}$ agrees with the measured one at one particular Reynolds number: choosing $R e=4.5$ leads in this way to the recently advanced angle $\theta_{c}^{a}=75^{\circ}$; choosing $R e=3.5$ leads to the recently receded one $\theta_{c}^{r}=36^{\circ}$. These values are in the range observed.

Contact-angle hysteresis is commonly attributed to both roughness and compositional heterogeneities of the solid surface (Johnson \& Dettre 1969), which can certainly exacerbate the underlying dynamics. In the present situation, both are present: the stainless steel surface shows grit marks that are visible to the naked eye; and the cleaning procedure described in $\$ 4.3$, although performed carefully, falls far short of the standards of surface chemistry. On the other hand, the surface is representative of real coating dies. Variations in contact angle may arise also when certain portions of the solid surface have been in contact with the liquid for longer times than others. This phenomenon, referred to as a 'kinetic effect' by Johnson \& Dettre (1969), most likely accounts for the difference in $\theta_{c}^{a}$ between data sets 1 and 3 in figure 23. To what extent the dynamic effects, i.e. slow relaxation of the contact line to its equilibrium position (see Teletzke et al. 1987), affect the experimental data is not clear. Waiting 20 instead of 5 minutes made differences that were smaller than the sensitivity of the measurements $(\sim 0.1 \mathrm{~mm})$.

The theoretical predictions and the measurements of contact line position in figure 24 agree well over a wide range of Reynolds number, even though contact angles are fitted at just one value of $R e$. In particular, the theoretical values of $R e_{L}$ and $R e_{V}$ at the turning points correspond quite closely to the flow rates at which the spontaneous flow transitions were observed in the experiment. Fluctuations in pumping rate and imperfect flow distributions across the width of the curtain probably account for some of what disagreement there is. A small discrepancy is that close to the upper turning 


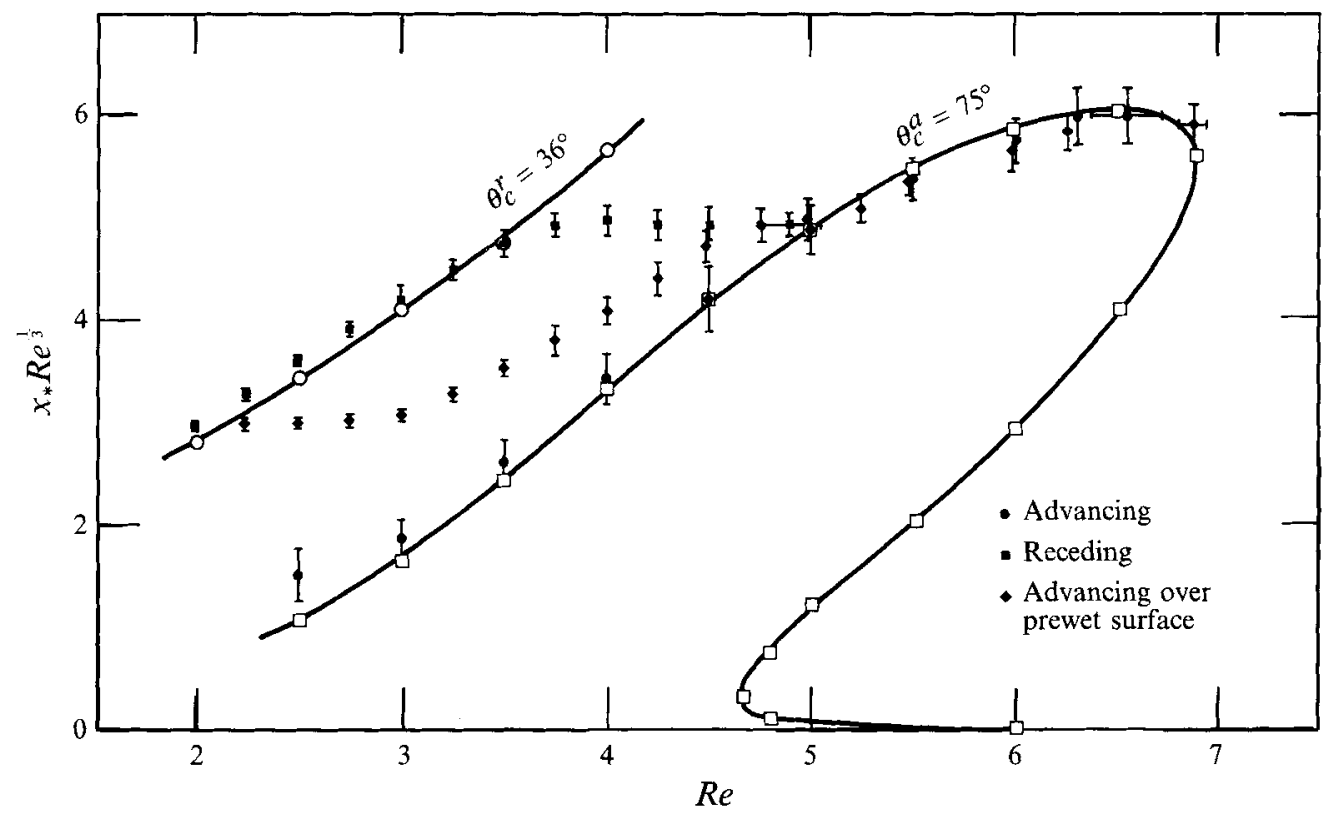

FIgURE 24. Hydrodynamic hysteresis and contact-angle hysteresis: finite-element predictions (open symbols) and experimental data of figure 23 (closed symbols) for $N_{\sigma} \approx 0.8, \beta=60^{\circ}, \gamma=60^{\circ}$.

point the predicted values of $x_{*} R e^{\frac{1}{3}}$ pass through a maximum, whereas in the experiment, as a consequence of contact-angle hysteresis, the contact line does not recede before it jumps.

The excellent match of experiment and theory in figure 24 argues for the validity of an important concept, namely that of a quasi-static apparent contact angle. Evidently, at a static contact line where a viscous free-surface flow separates from a solid surface and where the shape of the nearby meniscus, though controlled primarily by capillarity, can be influenced by viscous stress and inertial effects, the apparent contact angle is unaffected by major changes in the adjacent flow field. Similarly, the contactangle hysteresis at static contact lines with steady flow nearby appears to be, at least qualitatively, the same as in the absence of flow. In contrast, the 'dynamic' contact angle at a wetting 'line' where liquid continually encounters a moving surface is wellknown to be much affected by the adjacent flow field (e.g. Gutoff \& Kendrick 1982).

\subsection{Effects of wettability and lip design on hydrodynamic hysteresis}

A non-wetting surface on the lip underside should avert hydrodynamic hysteresis altogether. Common experience teaches that it is advantageous to coat the underside of a teapot spout with butter. In the experiments reported here, applying an adhesive Teflon tape to the lip underside did indeed almost completely prevent the contact line from advancing away from the edge.

Figure 25 shows how the hysteresis loop of contact line position versus Reynolds number contracts as the quasi-static contact angle $\theta_{c}$ is made larger. The range of $R e$ in which wetting phenomena do intrude diminishes as the lip underside is made less wetting. Furthermore, the maximum distance $x_{*}$ between the sharp edge and the contact line decreases. As $\theta_{c}$ comes near $98^{\circ}$, the two points approach one another and coalesce. At contact angles greater than $98^{\circ}$, the sharp edge inhibits spreading over the entire range of Reynolds numbers. This angle pertains, of course, only to the particular lip geometry $\left(\beta=60^{\circ}, \gamma=60^{\circ}\right)$ and liquid properties $\left(N_{\sigma}=1\right)$ chosen. Moreover, in 


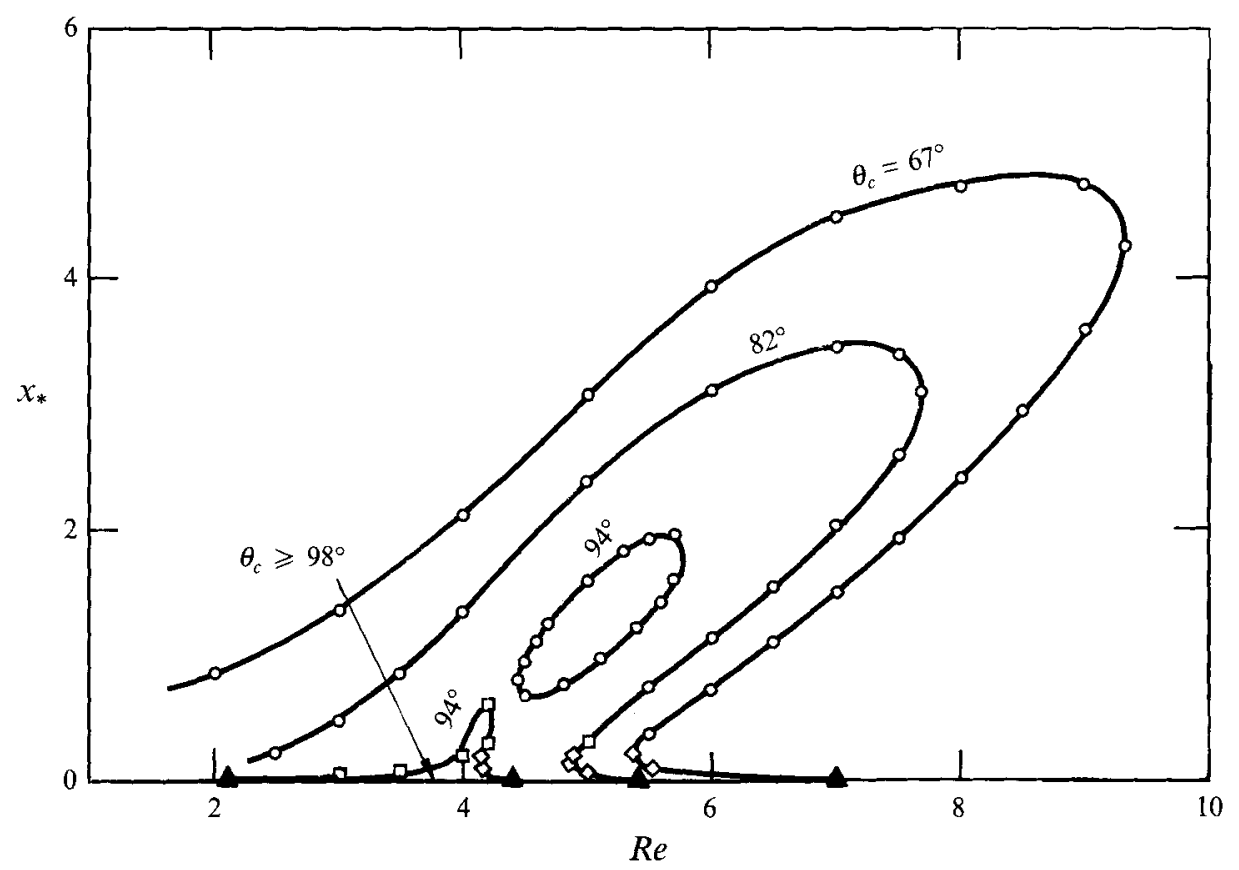

FIGURE 25. Family of hydrodynamic hysteresis loops with isolated solution branch $\left(N_{\sigma}=1, \beta=60^{\circ}\right.$, $\gamma=60^{\circ} ; \bigcirc$, tessellation type C; $\square$, type B with $N_{n}=7 ; \diamond$, type B with $N_{n}=5 ; \boldsymbol{\Delta}$, type A).

most real flow situations, a contact-angle hysteresis of the sort unveiled in figure 23 would be superimposed on the hydrodynamic hysteresis elucidated in figure 25 .

The critical angle $\theta_{c}^{\text {crit }}=98^{\circ}$ can be deduced from the Gibbs inequality condition (32) directly without computing complete hysteresis loops of the sort shown in figure 25. $\theta_{c}^{\text {crit }}=98^{\circ}$ is the angle that satisfies (32) when the critical separation angle $\theta_{s}^{\text {crit }}$ is taken to be that at the maximum of a curve of separation angle versus Reynolds number for the given lip configuration (cf. figure 15). Thus the role of the Gibbs inequality condition is twofold: it governs the conditions under which particular steady states with pinned contact line are realizable; and it constrains contact angles and lip configurations for which hydrodynamic hysteresis can be avoided altogether.

The hysteresis loop in figure 25 does not simply shrink and collapse into the abscissa as $\theta_{c}$ is increased. Analysis of the nonlinear equations and boundary conditions governing the film-forming flow reveals the existence of unexpected isolated solution branches. Such so-called isolas are common in many types of nonlinear systems (see Iooss \& Joseph 1980). Here, at a contact angle close to $94^{\circ}$, a closed loop of isolated steady states separates from the main hysteresis loop. Two turning points separate stable flow states on the upper half from unstable ones on the lower half. Stability is conjectured here from the way the solution family evolves. Experimentally, flow states on the isola cannot be reached by scanning flow rate, starting from either high or low rate, because the isola is disconnected from the main branch of states. They might be realized, however, if during operation in the range of Reynolds numbers covered by the isola, a large disturbance displaced the contact line sufficiently far away from the sharp edge. Special start-up procedures could guarantee this.

Contact line position is extremely sensitive to the inclination of the lip underside. Figure 26 reveals that tilting the lip underside downward from horizontal by only a few degrees makes the contact line advance a distance of a few film thicknesses. At 


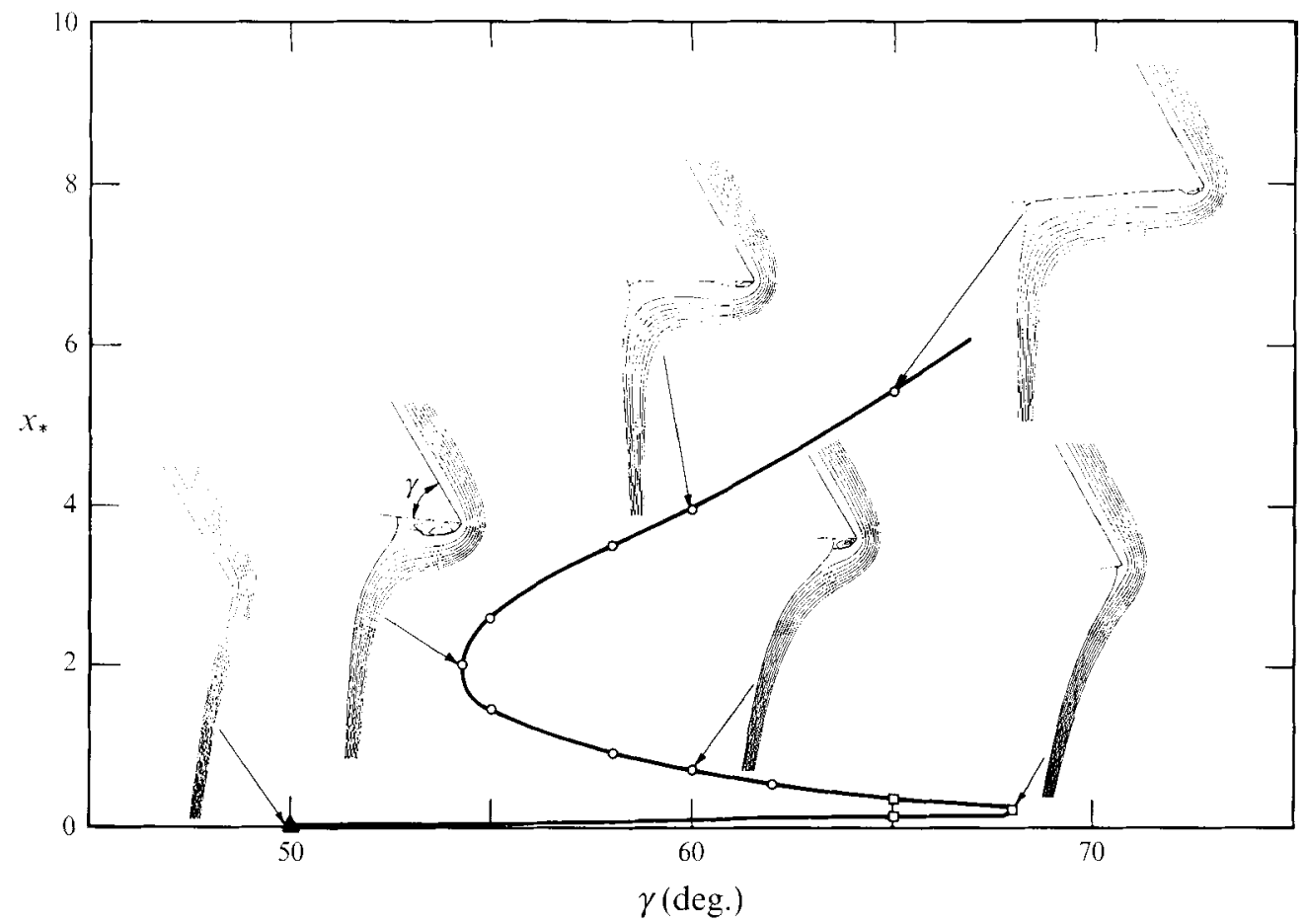

FIGURE 26. Hysteresis loop for contact line position $x_{*}$ as a function of cut-back angle $\gamma(\operatorname{Re}=6$, $N_{\sigma}=1, \theta_{c}=67^{\circ}, \beta=60^{\circ} ; O$, tessellation type C; $\square$, type B; $\boldsymbol{\Delta}$, type A).

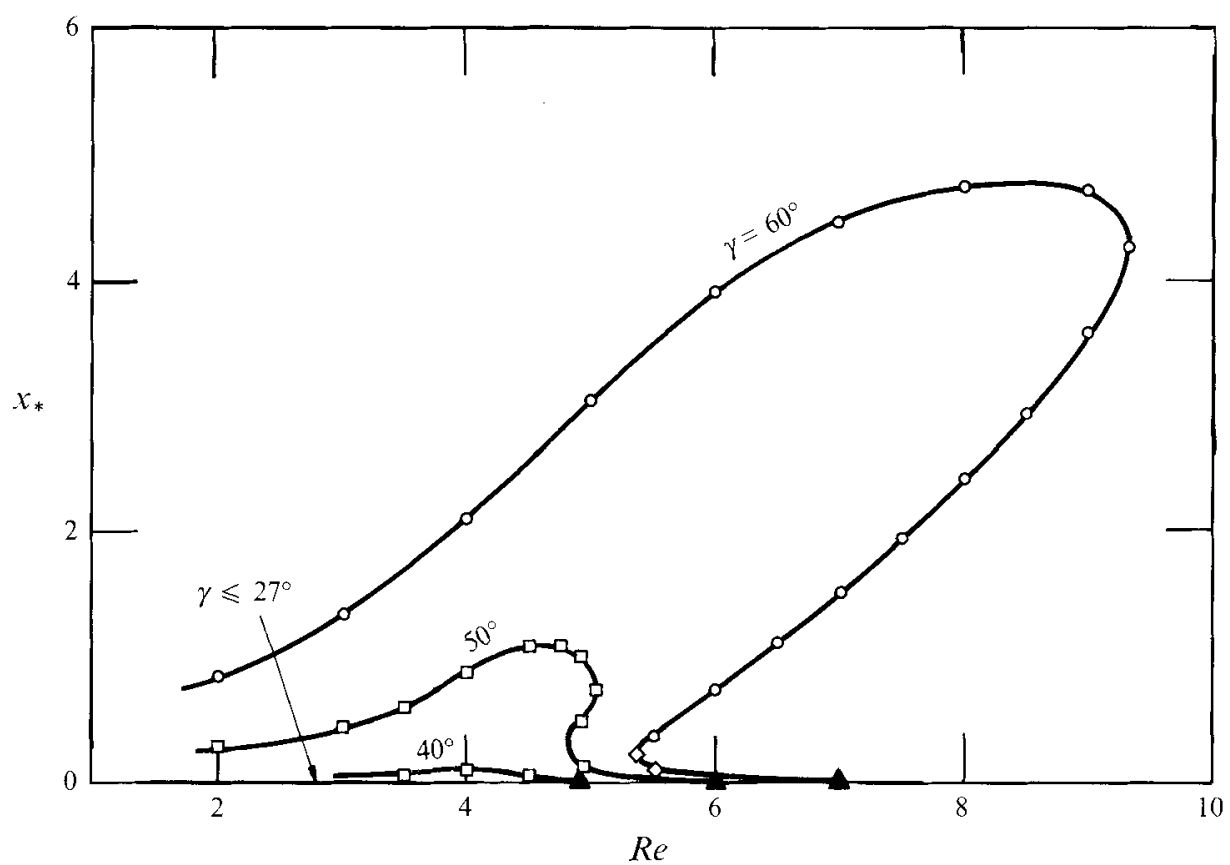

FIGURF 27. Disappearance of hydrodynamic hysteresis loop with decreasing cut-back angle $\gamma$ $\left(N_{r}=1, \theta_{c}=67^{\circ}, \beta=60^{\circ} ; \bigcirc\right.$, tessellation type C; $\square$, type B with $N_{n}=7 ; \diamond$, type B with $N_{n}=5$; $\Delta$, type A). 


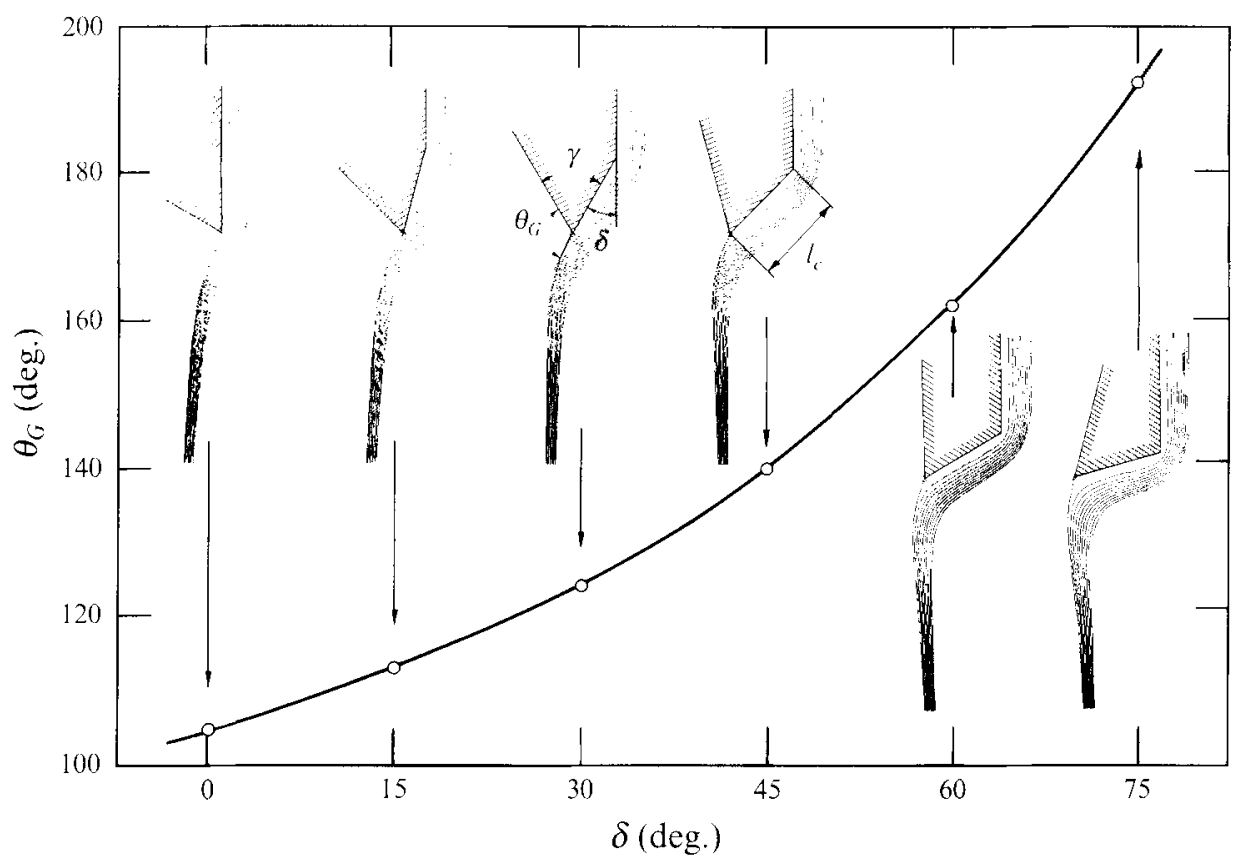

Figurf 28. Finite-element predictions of sheet-forming flows with improved lip design $\left(R e=5, N_{\tau}=1.15, \beta=60^{\circ}, \gamma=60^{\circ}\right)$.

sufficiently large $\gamma$, of course, the liquid film attaches completely to the solid instead of falling off as an unsupported curtain. On the other hand, tilting the lip underside upward from horizontal, by only $10^{\circ}$ at the particular set of flow parameters considered here, ensures that the contact line remains pinned at the sharp corner. The curve of $x_{*}$ vs. $\gamma$ at constant $R e$ forms another hysteresis loop, as might be expected, for it is just another section through a surface of states in the $\left(x_{*}, R e, \gamma\right)$-space at fixed $N_{\sigma}$ and $\theta_{r}$.

The results in figure 26 imply that the hydrodynamic hysteresis is largely due to the relative ease with which the contact line can migrate in a direction almost normal to gravity when the lip underside is close to being horizontal. When $\gamma$ is much larger than $60^{\circ}$ (at $\beta=60^{\circ}$ ), the hysteresis loop of $x_{*} v s$. Re expands beyond all bounds. At all but extremely high Reynolds numbers, the only stable states are those in which the liquid dribbles along the lip underside. When, on the other hand, the lip is made sharper, the loop shrinks rapidly as shown in figure 27 . At the particular set of parameters considered, a cut-back angle smaller than $27^{\circ}$ ensures that the contact line remains pinned over the entire range of Reynolds numbers.

These results rationalize another strategy for making sure that wetting phenomena and associated hysteresis effects do not intrude. The cut-back angle $\gamma$ between the two solid surfaces ought to be made as acute as other design criteria permit. Moreover, a horizontal lip underside is to be avoided. Indeed, choosing $\beta-\gamma$ as large as possible appears best. This criterion is put to work in the improved lip design shown in figure 28 (Dittman et al. 1976 disclosed similar lip configurations): the lowest portion of the lip is cut back at an angle $\delta$, but the angle $\gamma$ at the corner where the free surface separates from the solid is held fixed at $60^{\circ}$. The distance $l_{c}$ between the two corners of the solid is an additional design parameter; $l_{c}=3$ is taken in the present case study.

Cutting back the lip yields two benefits. First, the hydrodynamic deflection almost 

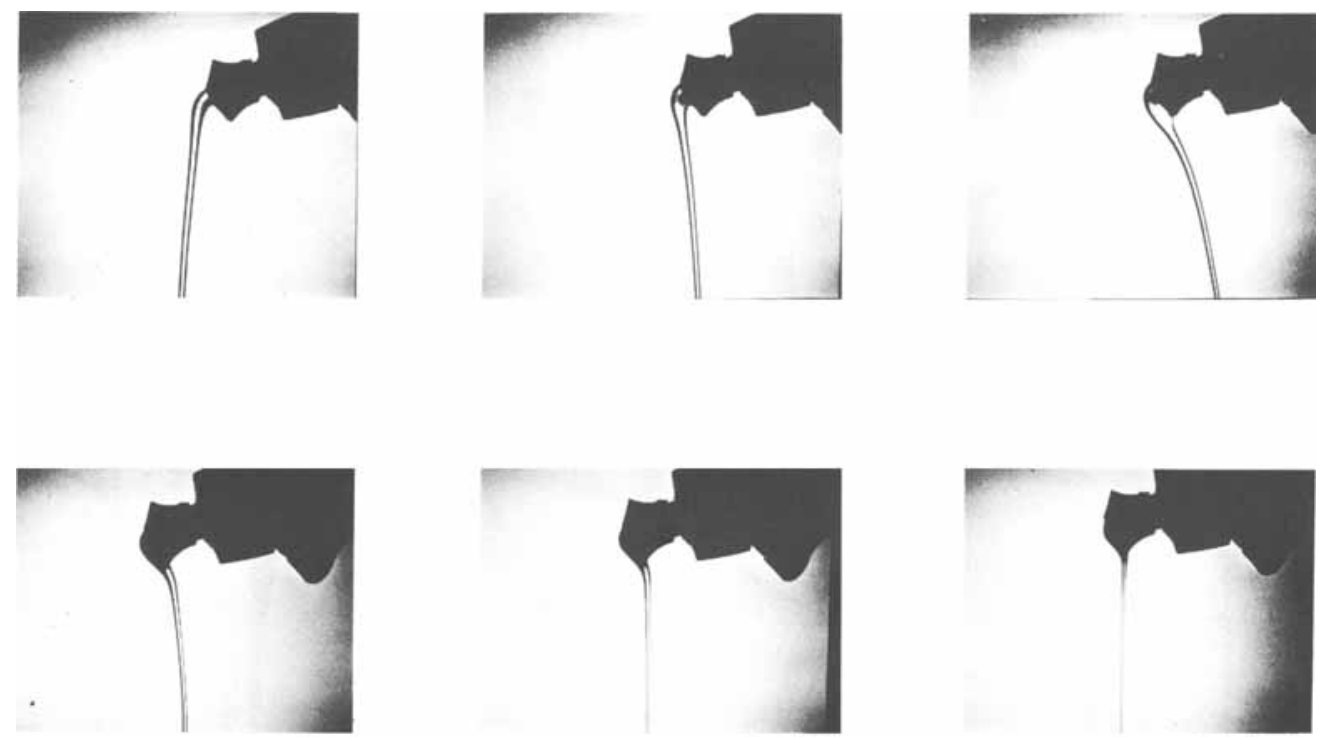

FIGURE 29. Detergent flowing from the spout of a commercial dispensing bottle (flow rate decreases from top left to bottom right).

disappears as the angle $\delta$ is made larger. Second, the separation angle $\theta_{G} \equiv\left(180^{\circ}-\gamma\right)+\left(180^{\circ}-\theta_{s}\right)$ on the gas side increases and eventually exceeds $180^{\circ}$. The Gibbs inequality condition (32) implies that when $\theta_{G}>180^{\circ}$ the contact line does not advance whatever the contact angle appears to be. At very small contact angles, however, the contact line might recede to another position between the two corners of the solid. Furthermore, the design study in figure 28 pertains to just one particular set of flow parameters. Thus a single lip design is unlikely to be optimal over wide ranges of flow parameters and contact angles.

The spout design of certain commercial detergent bottles, such as the one shown in figure 29 , mimics closely the modified lip design suggested in figure 28 , even though the original purpose of that spout design might have been quite different. Observation reveals that, indeed, the detergent rarely wets the underside of the spout. And in everyday life one finds a great variety of old and new teapot designs, some of which perform well and others not - for reasons elucidated in this paper.

\section{Concluding remarks}

This study establishes a comprehensive theory for sheet-forming flow off the lip of an inclined plane, a flow that had been locked away from analysis. The theory rests on combining Galerkin's weighted residual technique with convenient finite-element basis functions, spine parametrization, and isoparametric mapping to solve efficaciously the equations of steady two-dimensional viscous free-surface flow. Computational methods for viscous free-surface flow analysis are still evolving, but the developments put forward by Kistler \& Scriven $(1983,1984)$ have proven particularly advantageous for the analysis reported in this paper. Not only has the computer-aided analysis produced complete descriptions of the flow fields and free-surface shapes of interest; it has also led, in combination with corroborating experiments, to a thorough understanding of the basic mechanisms that cause the teapot effect. Nonetheless, the intricate structure of the solution space uncovered points toward the value of 
incorporating, in finite-element algorithms of the sort summarized in $\$ 2$, more efficient schemes for tracking the evolution. multiplicity, and stability of viscous free-surface flows. Such schemes might include arclength continuation with adaptive, automatic parameter step control (cf. Kheshgi et al. 1983), as well as a strategy to automatically trace loci of turning points in solution space (cf. Christodoulou \& Scriven 1994).

The mechanisms behind the teapot effect can be summarized as follows. The deflection of the liquid results from the interplay of purely hydrodynamic forces, in particular the lack of shear in the sheet as it falls away from the edge of the lip (Rubin \& Wharshavsky 1970 report an experimental observation that the 'antiballistic' deflection is influenced by polymeric additives, an effect to be investigated further). The Gibbs inequality condition rules under what conditions the contact line actually remains pinned at the sharp edge. When it does not, with the result that wetting and spreading phenomena intrude, the flow field, free surfaces, and contact line depend in quite complex and unexpected ways on flow parameters, lip shape, and wettability. Hysteresis arises both because of purely hydrodynamic effects, i.e. multiple steady-state configurations at identical flow parameters and apparent contact angle, and because of surface effects, i.e. contact-angle hysteresis, particularly that associated with surface roughness and heterogeneity. Steady flow states on isolas further complicate the structure of the space of solutions of the system of governing equations. Thus the teapot effect is more than merely an issue of wetting as one might be led to believe by its annoying everyday occurrence.

Beyond explaining the teapot effect, the results of this study point to conclusions of some generality. One is that in macroscopic analysis of viscous free-surface flows, the sub-microscopic details of the physics at a static contact line can be ignored. The concept of a quasi-static apparent contact angle $\theta_{c}$ is entirely adequate, provided the underlying dynamics of equilibration are either very rapid or very slow compared to the time interval in which the flow is operated. That angle shows in essence the same behaviour as the observed contact angle at a contact line where a meniscus that bounds the same liquid at rest appears to intersect the same solid surface: $\theta_{c}$ is not affected by appreciable flow nearby, nor by substantial changes in the adjacent flow field; $\theta_{c}$ exhibits contact-angle hysteresis between recently advanced and recently receded configurations; and $\theta_{c}$ enters the Gibbs inequality condition that quantifies the inhibiting effect of a sharp edge on spreading of liquid, whether or not there is flow nearby. Of course, if the apparent contact angle is to be inferred from first principles, the physics of wetting at submicroscopic scales has to be resolved (see Benner, Scriven \& Davis 1983; Teletzke et al. 1987, 1988).

Perhaps the most striking result of this study is that viscous free-surface flows can exhibit multiple steady states that are associated with a static separation line yet are completely independent of the contact-angle hysteresis. Similar hysteresis effects ought to affect contact line position in many other coating and polymer processing flows where a free surface separates from a solid near a sharp edge. Understanding this hysteresis is important, for it demonstrates that the observed flow, and therewith possibly product quality, depend not only on flow parameters, wettability, and die configurations, but sometimes also on the way the flow is started up. For the case of falling liquid curtains, that understanding has led to identification and evaluation of different ways of avoiding the deleterious consequences of the teapot effect.

C. A. Dowd contributed significantly to the experimental part of this work. The $3 \mathrm{M}$ Company made available key components of the experimental set-up, for which we are grateful. Financial support was provided early by the Fluid Mechanics Program of the 
National Science Foundation, and later by grants-in aid from 3M Company, Xerox Corporation, Eastman Kodak Company, Fuji Photo Film Co. Ltd., and Polaroid Foundation, by the University of Minnesota Computer Center, and by a University of Minnesota Graduate School Fellowship. For valuable discussions we are indebted to O. A. Basaran, R. E. Benner, D. J. Coyle, H. T. Davis, H. S. Kheshgi, P. M. Schweizer, G. S. Sterzi and G. F. Teletzke.

\section{REFERENCES}

Aввотт, J.P. 1978 An efficient algorithm for the determination of certain bifurcation points. J. Comput. Appl. Math. 4, 19-26.

Adamson, A. W. 1982 Physical Chemistry of Surfaces, 4th edn. Wiley.

Benner, R. E., Scriven, L. E. \& Davis, H. T. 1982 Structure and stress in the gas-liquid-solid contact region. In Structure of the Interfacial Region (ed. M. Lal). Faraday Symp. No. 16, pp. 169-190. The Royal Society of Chemistry, London.

Bretherton, F. P. 1961 The motion of long bubbles in tubes. J. Fluid Mech. 10, 166-188.

BRown, D. R. 1961 A study of the behaviour of a thin sheet of moving liquid. J. Fluid Mech. 10, 297-305.

Chang, P. W., Patten, T. W. \& Finlayson, B. A. 1979 Collocation and Galerkin finite element methods for viscoelastic fluid flow - II. Die swell problem with a free surface. Comput. Fluids 7 , 285-303

Christodoulou, K. N. \& Scriven, L. E. 1994 Operability limits of free surface flow systems by solving nonlinear eigenvalue problems. Intl J. Num. Meth. Fluids (to appear).

Clarke, N. S. 1968 Two-dimensional flow under gravity in a jet of viscous liquid. J. Fluid Mech. 31, 481-500.

Cruickshank, J. O. \& Munson, B. R. 1981 Viscous buckling of plane and axisymmetric jets. J. Fluid Mech. 113, 221-239.

Cullen, E. J. \& Davidson, J. F. 1957 Absorption of gas in liquid jets. Trans. Faraday Soc. 53, $113-120$.

Dittmann, D. A., Griswold, G. L., Wright, S. E. \& Winkler, D. E. 1976 Curtain coating apparatus. Research Disclosure 147, 19 (No. 14715, July 1976).

DUPRET, F. 1982 A method for the computation of viscous flow by finite elements with free boundaries and surface tension. In Finite Element Flow Analysis, Proc. Fourth Intl Conf. on Finite Element Methods in Flow Problems Tokyo, 26-29 July 1982 (ed. T. Kawai), pp. 495-502. University of Tokyo Press.

Gear, R. L. Keentok, M., Milthorpe, J. F. \& Tanner, R. I. 1983 The shape of low Reynolds number jets. Phys. Fluids. 26, 7-25.

GibBs, J. W. 1906 The Scientific Papers of J. Willard Gibbs. Vol. I: Thermodynamics. Longmans Green \& Co. (Dover Reprint, 1961).

GrFFoRd, W. A. 1982 A finite element analysis of isothermal fiber formation. Phys. Fluids 25, 219-225.

Grelller, G. F. 1972 Method for Making Photographic Elements. US Patent No. 3,632,374.

Gutoff, E. B. \& Kendrick, C. E. 1982 Dynamic contact angles. AIChE J. 28, 459-466.

Harlow, F. H. \& Amsden, A. A. 1971 Fluid Dynamics. Los Alamos Scientific Laboratory Monograph LA-4700.

Higgins, B. G. \& SCRIVEN, L. E. 1979 Interfacial shape and evolution equations for liquid films and other viscocapillary flows. I\&EC Fundam. 18, 208-215.

Hirt, C. W. \& Shannon, J. P. 1968 Free surface stress conditions for incompressible flow calculations. J. Comput. Phys, 2, 403-411.

Hood, P. 1976 Frontal solution program for unsymmetric matrices. Intl J. Num. Meth. Engng 10, 379-393; and Correction. Intl J. Num. Meth. Engng. 11, (1977) 1055.

Hur, C. \& Mason, S. G. 1977 Effects of surface roughness on wetting. J. Colloid Interface Sci. 60, $11-38$.

Huyakorn, P. S., Taylor, C., Lee, R. L. \& Gresho, P. M. 1978 A comparison of various mixed 
interpolation finite elements in the velocity-pressure formulation of the Navier-Stokes equations. Comput. Fluids 6, 25-35.

Iooss, G. \& JosePH, D. D. 1980 Elementary Stability and Bifurcation Theory. Springer.

Johnson, R. E. \& DetTre, R. H. 1969 Wettability and contact angles. In Surface and Colloid Science (ed. E. Matijevic), Vol. 2, pp. 85-153. Wiley Interscience.

JoSePh, D. D., NGyen, K. \& MatTA, J. E. 1983 Jets into liquid under gravity. J. Fluid Mech. 128, $443-468$.

Keller, H. B. 1977 Numerical solution of bifurcation and non-linear eigenvalue problems. In Applications in Bifurcation Theory (ed. P. H. Rabinowitz), pp. 359-384. Academic.

Keller, J. B. 1957 Teapot effect. J. Appl. Phys. 28, 859-864.

Kheshgi, H. S., Basaran, O. A., Benner, R. E., Kistler, S. F. \& Scriven, L. E. 1983 Continuation in a parameter: experience with viscous and free surface flows. In Numerical Methods in Laminar and Turbulent Flow, Proc. Third. Intl Conf. on Finite Element Methods in Flow Problems, Seattle 8-11 August 1983 (ed. C. Taylor et al.). Pineridge Press.

KISTLER, S. F. 1984 The fluid mechanics of curtain coating and related viscous free surface flows with contact lines. PhD dissertation, University of Minnesota, Minneapolis, USA.

Kistler, S. F. \& SCriven, L. E. 1983 Coating flows. In Computational Analysis of Polymer Processing (ed. J. R. A. Pearson \& S. M. Richardson), pp. 243-299. Applied Science Publishers.

Kistrer, S. F. \& SCRIVEN, L. E. 1984 Coating flow theory by finite element and asymptotic analysis of the Navier-Stokes system. Intl J. Num. Meth. Fluids 4, 207-229.

LiN, S. P. 1981 Stability of a falling curtain. J. Fluid Mech. 104, 111-118.

Lin, S. P. \& Krishna, M. V. G. 1978 Deflection of a viscous liquid curtain. Phys. Fluids 21, 2367-2368.

Martin, H. R. \& Friedman, S. B. 1974 A review of research related to the development of liquid jet fluidics. Fluidics Q. 6, 67-79.

Michael, D. H. 1958 The separation of a viscous liquid at a straight edge. Mathematika 5, 82-84.

Mohanty, K. K. 1981 Fluid in porous media: two-phase distribution and flow. PhD dissertation, University of Minnesota, Minneapolis, USA.

Newman, A. A. 1968 Glycerol. Morgan-Grampian.

Nickell, R. E., TANNER, R. I. \& Caswell, B. 1974 Solution of viscous incompressible jet and free surface flows using finite element method. J. Fluid Mech. 65, 189-206.

Novy, R. A., DAvis, H. T. \& SCRIVEN, L. E. 1990 Upstream and downstream boundary conditions for continuous-flow systems. Chem. Engng Sci. 45, 1515-1524.

OGaWA, S. \& SCRIVEN, L. E. 1994 An analysis of curtain coating flow. AIChE J. (in press).

Oliver, J. F., Huh, C. \& Mason, S. G. 1977 Resistance to spreading of liquids by sharp edges. J. Colloid Interface Sci. 59, 568-581.

OLrver, J. F., Huh, C. \& MASON, S. G. 1980 Experimental study of some effects of solid surface roughness on wetting. Colloids and Surfaces 1, 79-90.

PRITCHARD, W. G. 1986 Instability and chaotic behaviour in a free surface flow. J. Fluid Mech. 165, $1-60$.

RauX, R. 1976 Appareil de Revêtement par Rideau Liquide. Brevet d'Invention 833.851, Royaume de Belgique.

Reiner, M. 1956 The teapot effect. Physics Today 9, 16-20 (September).

ReIner, M. 1969 Deformation, Strain and Flow, 3rd edn. H. K. Lewis \& Co.

Richardson, S. 1970 The die swell phenomenon. Rheol. Acta 9, 193-199.

RuBin, H. \& WhaRSHAVSKy, M. 1970 A note on the break-up of viscoelastic liquid jets. Israel J. Technol. 8, 285-288.

RUSCHAK, K. J. 1978 Flow of a falling film into a pool. AIChE J. 24, 705-709.

RUSCHAK, K. J. 1980 A method for incorporating free boundaries with surface tension in finite element fluid flow simulators. Intl J. Num. Meth. Engng 15, 639-648.

SaIto, H. \& SCRIVEn, L. E. 1981 Study of coating flow by the finite element method. J. Comput. Phys. $42,53-76$.

Silliman, W. J. \& Scriven, L. E. 1980 Separating flow near a static contact line: slip at wall and the shape of the free surface. J. Comput. Phys. 34, 287-313. 
TAYLOR, G. I. 1968 Instability of jets, threads and sheets of viscous fluids. In Proc. Intl Congr. Appl. Mech. Stanford 1968 (ed. M. Hetenyi \& W. G. Vicenti), p. 382. Springer.

Teletzke, G. F., Davis, H. T. \& Scriven, L. E. 1987 How liquids spread on solids. Chem. Engng Commun. 55, 41-81.

Teletzke, G. F., Davis, H. T. \& Scriven, L. E. 1988 Wetting hydrodynamics. Rev. Phys. Appl. 23, 989-1007.

Vanden-Broeck, J.-M. \& Keller, J. B. 1986 Pouring flows. Phys. Fluids 29, 3958-3961.

Vanden-Broeck, J.-M. \& Keller, J. B. 1988 Pouring flows with separation. Phys. Fluids A 1, $156-158$.

WALKER, J. 1984 The troublesome teapot effect, or why a poured liquid clings to the container. Scientific American 251, 144-152 (October).

Walters, R. A. 1980 The frontal method in hydrodynamic simulations. Comput. Fluids 8, 265-272. 